MATHEMATICS OF COMPUTATION

Volume 75 , Number 253, Pages 395-417

S 0025-5718(05)01784-9

Article electronically published on September 29, 2005

\title{
SOME REMARKS ON RICHARDSON ORBITS IN COMPLEX SYMMETRIC SPACES
}

\author{
ALFRED G. NOËL
}

\begin{abstract}
Roger W. Richardson proved that any parabolic subgroup of a complex semisimple Lie group admits an open dense orbit in the nilradical of its corresponding parabolic subalgebra. In the case of complex symmetric spaces we show that there exist some large classes of parabolic subgroups for which the analogous statement which fails in general, is true. Our main contribution is the extension of a theorem of Peter E. Trapa (in 2005) to real semisimple exceptional Lie groups.
\end{abstract}

\section{INTRODUCTION}

In this paper, unless otherwise specified, $\mathfrak{g}$ will be a real semisimple Lie algebra with adjoint group $G$ and Cartan decomposition $\mathfrak{g}=\mathfrak{k} \oplus \mathfrak{p}$ relative to a Cartan involution $\theta$. We will denote by $\mathfrak{g}_{\mathbb{C}}$ the complexification $\mathfrak{g}$. Then $\mathfrak{g}_{\mathbb{C}}=\mathfrak{k}_{\mathbb{C}} \oplus \mathfrak{p}_{\mathbb{C}}$, where $\mathfrak{k}_{\mathbb{C}}$ and $\mathfrak{p}_{\mathbb{C}}$ are obtained by complexifying $\mathfrak{k}$ and $\mathfrak{p}$, respectively. $K$ will be a maximal compact Lie subgroup of $G$ with Lie algebra $\mathfrak{k}$, and $K_{\mathbb{C}}$ will be the connected subgroup of the adjoint group $G_{\mathbb{C}}$ of $\mathfrak{g}_{\mathbb{C}}$, with Lie algebra $\mathfrak{k}_{\mathbb{C}}$. It is well known that $K_{\mathbb{C}}$ acts on $\mathfrak{p}_{\mathbb{C}}$ and the number of nilpotent orbits of $K_{\mathbb{C}}$ in $\mathfrak{p}_{\mathbb{C}}$ is finite. Furthermore, for a nilpotent $e \in \mathfrak{p}_{\mathbb{C}}, K_{\mathbb{C}} \cdot e$ is a connected component of $G_{\mathbb{C}} \cdot e \cap \mathfrak{p}_{\mathbb{C}}$.

Let $\mathfrak{q}$ be a parabolic subalgebra of $\mathfrak{g}_{\mathrm{c}}$ with Levi decomposition $\mathfrak{q}=\mathfrak{l} \oplus \mathfrak{u}$. Denote by $Q$ the connected Lie subgroup of $G_{\mathbb{C}}$ with Lie algebra $\mathfrak{q}$. Then there is a unique orbit $\mathcal{O}_{\mathfrak{g}_{\mathbb{C}}}$ of $G_{\mathbb{C}}$ on $\mathfrak{g}_{\mathbb{C}}$ meeting $\mathfrak{u}$ in an open dense set. The intersection $\mathcal{O}_{\mathfrak{g}_{\mathbb{C}}} \cap \mathfrak{u}$ consists of a single $Q$-orbit under the adjoint action of $Q$ on $\mathfrak{u}$. These facts were first proved by Richardson $\left[5\right.$. Hence, $\mathcal{O}_{\mathfrak{g}_{\mathbb{C}}}$ is called a Richardson orbit. Since the publication in 1979 of a fundamental paper of Lusztig and Spaltenstein [3, relating Representation Theory of $\mathfrak{g}_{\mathbb{C}}$ to Richardson orbits mathematicians have paid a lot of attention to such orbits. However, most of the work was done for complex semisimple Lie groups. Lately, after the proof of the Kostant-Sekiguchi correspondence [6], some initiatives have been taken to study Richardson orbits of real Lie reductive groups.

The Kostant-Sekiguchi correspondence is a bijection between nilpotent orbits of $G$ in $\mathfrak{g}$ and nilpotent orbits of $K_{\mathbb{C}}$ on $\mathfrak{p}_{\mathbb{C}}$. Thus, the correspondence allows us to

Received by the editor March 15, 2004.

2000 Mathematics Subject Classification. Primary 17B05, 17B10, 17B20, 22 E30.

Key words and phrases. Parabolic group, nilpotent orbits, prehomogeneous spaces.

The author was partially supported by an NSF research opportunity award sponsored by David Vogan of MIT. He thanks him for the support. The author is also grateful to Donald R. King and Peter E. Trapa for several discussions about the content of this paper. Finally, he expresses his thanks to the referee for his kind words.

(C)2005 American Mathematical Society Reverts to public domain 28 years from publication 
study certain questions about real nilpotent orbits by looking at nilpotent orbits of $K_{\mathbb{C}}$ on the symmetric space $\mathfrak{p}_{\mathbb{C}}$. Therefore the following is a natural question: Maintaining the above notation and assuming that $\mathfrak{q}$ is $\theta$-stable, when does $Q \cap K_{\mathrm{C}}$ admit an open dense orbit on $\mathfrak{u} \cap \mathfrak{p}_{\mathbb{C}}$ ? This would be the equivalent of Richardson's theorem for the real case. It turns out that this statement is not true in general. Patrice Tauvel gave a counterexample in [7, p. 652] for $\mathfrak{g}_{\mathrm{C}}=D_{4}$. However, he was able to prove an interesting version of Richardson's theorem. We shall say more about this later. In the next section we shall give some important cases where the equivalent of Richardson's theorem still holds.

\section{Some Density RESUlts}

2.1. Jacobson-Morozov parabolic subalgebra. Let $(x, e, f)$ to be a normal $\mathfrak{s l}_{2}$-triple with $x \in i \mathfrak{k} e$ and $f \in \mathfrak{p}_{\mathbb{C}}$. From the representation theory of $\mathfrak{s l}_{2}, \mathfrak{g}_{\mathbb{C}}$ has the following eigenspace decomposition:

$$
\mathfrak{g}_{\mathbb{C}}=\bigoplus_{j \in \mathbb{Z}} \mathfrak{g}_{\mathbb{C}}^{(j)}, \quad \text { where } \mathfrak{g}_{\mathbb{C}}^{(j)}=\left\{z \in \mathfrak{g}_{\mathbb{C}} \mid[x, z]=j z\right\} .
$$

The subalgebra $\mathfrak{q}=\bigoplus_{j \in \mathbb{N}} \mathfrak{g}_{\mathbb{C}}^{(j)}$ is a parabolic sub algebra of $\mathfrak{g}_{\mathbb{C}}$ with a Levi part $\mathfrak{l}=\mathfrak{g}_{\mathrm{C}}^{(0)}$ and nilradical $\mathfrak{u}=\bigoplus_{j \in \mathbb{N}^{*}} \mathfrak{g}_{\mathbb{C}}^{(j)}$.

Call $\mathfrak{q}$ the Jacobson-Morosov parabolic subalgebra of $e$ relative to the triple $(x, e, f)$. Our choice of the triple $(x, e, f)$ forces $\mathfrak{q}$ to be $\theta$-stable in Vogan's sense.

Retain the above notation. Let $Q$ be the connected subgroups of $G_{\mathrm{C}}$ with Lie algebra $\mathfrak{q}$. We shall prove that if $e$ is an even nilpotent, then Richardson's theorem holds on $\mathfrak{u} \cap \mathfrak{p}_{\mathbb{C}}$.

Let $\mathfrak{q}$ be the Jacobson-Morozov parabolic subalgebra of $e$ relative to the normal triple $(x, e, f)$. Then

Proposition 2.1. $Q \cap K_{\mathbb{C}}$.e is a dense open subset of $\bigoplus_{i \geq 2} \mathfrak{g}_{\mathbb{C}}^{(i)} \cap \mathfrak{p}_{\mathbb{C}}$. Moreover if $e$ is even, that $i s, \mathfrak{g}_{\mathbb{C}}^{(i)}=0$ for $i$ odd, then $\overline{Q \cap K_{\mathbb{C}} \cdot e}=u \cap \mathfrak{p}_{\mathbb{C}}$.

Proof. It is a result of Carter ([1, Proposition 5.7.3]) that the orbit of $Q$ on $\bigoplus_{i \geq 2} \mathfrak{g}_{\mathbb{C}}^{(i)}$ containing $e$ is a dense open subset of $\bigoplus_{i \geq 2} \mathfrak{g}_{\mathbb{C}}^{(i)}$. It follows that $[\mathfrak{q}, e]=\bigoplus_{i \geq 2} \mathfrak{g}_{\mathbb{C}}^{(i)}$ which implies that

$$
[\mathfrak{q}, e]=\left[\mathfrak{q} \cap \mathfrak{k}_{\mathbb{C}}, e\right] \oplus\left[\mathfrak{q} \cap \mathfrak{p}_{\mathbb{C}}, e\right] .
$$

Since each $\mathfrak{g}_{\mathrm{C}}^{(i)}$ is $\theta$-stable,

$$
\bigoplus_{i \geq 2} \mathfrak{g}_{\mathbb{C}}^{(i)}=\bigoplus_{i \geq 2} \mathfrak{g}_{\mathbb{C}}^{(i)} \cap \mathfrak{k}_{\mathbb{C}} \oplus \mathfrak{g}_{\mathbb{C}}^{(i)} \cap \mathfrak{p}_{\mathbb{C}}
$$

The fact that $e \in \mathfrak{p}_{\mathbb{C}}$ and the previous direct sum decomposition force

$$
\left.\mathfrak{q} \cap \mathfrak{k}_{\mathbb{C}}, e\right]=\bigoplus_{i \geq 2} \mathfrak{g}_{\mathbb{C}}^{(i)} \cap \mathfrak{p}_{\mathbb{C}}
$$

Hence, the map $a d_{e}: \mathfrak{q} \cap \mathfrak{k}_{\mathbb{C}} \rightarrow \bigoplus_{i \geq 2} \mathfrak{g}_{\mathbb{C}}^{(i)} \cap \mathfrak{p}_{\mathbb{C}}$ is surjective.

It is clear that $\bigoplus_{i \geq 2} \mathfrak{g}_{\mathbb{C}}^{(i)} \cap \mathfrak{p}_{\mathbb{C}}$ is a $Q \cap K_{\mathbb{C}}$-module under the adjoint action. Also $e \in \mathfrak{g}_{\mathbb{C}}^{(2)} \cap \mathfrak{p}_{\mathbb{C}} \subseteq \bigoplus_{i \geq 2} \mathfrak{g}_{\mathbb{C}}^{(i)} \cap \mathfrak{p}_{\mathbb{C}}$. Thus the map $z \rightarrow A d_{z}(e)$ is a morphism from $Q \cap K_{\mathbb{C}}$ to $\bigoplus_{i \geq 2} \mathfrak{g}_{\mathbb{C}}^{(i)} \cap \mathfrak{p}_{\mathbb{C}}$ and its differential is the map $-a d_{e}: \mathfrak{q} \cap \mathfrak{k}_{\mathbb{C}} \rightarrow \bigoplus_{i \geq 2} \mathfrak{g}_{\mathbb{C}}^{(i)} \cap \mathfrak{p}_{\mathbb{C}}$. 
This map is surjective. Thus the given morphism is dominant and separable. Since the image of such a map is open in its closure, the $Q \cap K_{\mathbb{C}}$-orbit of $e$ is a nonempty open dense subset of $\bigoplus_{i \geq 2} \mathfrak{g}_{\mathbb{C}}^{(i)} \cap \mathfrak{p}_{\mathbb{C}}$.

If $e$ is even, then $\bigoplus_{i>2} \mathfrak{g}_{\mathbb{C}}^{(i)} \cap \mathfrak{p}_{\mathbb{C}}=u \cap \mathfrak{p}_{\mathbb{C}}$. Hence, $\overline{Q \cap K_{\mathbb{C}} \cdot e}=u \cap \mathfrak{p}_{\mathbb{C}}$.

2.2. Borel-de Siebenthal parabolic subalgebras. A complex Lie algebra $\mathfrak{g}_{\mathbb{C}}$ is said to be graded if $\mathfrak{g}_{\mathbb{C}}=\bigoplus_{k=-\infty}^{\infty} \mathfrak{g}_{\mathbb{C}}^{k}$, where $\mathfrak{g}_{\mathbb{C}}^{k}$ is a vector subspace of $\mathfrak{g}_{\mathbb{C}}$ and $\left[\mathfrak{g}_{\mathbb{C}}^{i}, \mathfrak{g}_{\mathbb{C}}^{j}\right]=\mathfrak{g}_{\mathbb{C}}^{i+j}$ for all integers $i$ and $j$.

We shall need the following theorem of Vinberg:

Theorem 2.2. Let $G_{\mathbb{C}}$ be a complex semisimple Lie group with graded Lie algebra $\mathfrak{g}_{\mathbb{C}}=\bigoplus_{k} \mathfrak{g}_{\mathbb{C}}^{k}$, and let $G_{\mathbb{C}}^{0}$ be the analytic subgroup of $G_{\mathbb{C}}$ with Lie algebra $\mathfrak{g}_{\mathbb{C}}^{0}$. Then the adjoint action of $G_{\mathbb{C}}^{0}$ on $\mathfrak{g}_{\mathbb{C}}^{1}$ has only finitely many orbits. Hence one of them must be open.

Proof. See Vinberg [9].

A proof of the uniqueness and denseness of such an open orbit is found in Knapp [2, Proposition 10.1].

Let $\mathfrak{g}$ be of inner-type, that is, $\operatorname{rank}(\mathfrak{k})=\operatorname{rank}(\mathfrak{g})$, and let $\Delta$ be a $\operatorname{Vogan} 11$ set of simple roots of $\mathfrak{g}_{\mathbb{C}}$. Then $\Delta$ can be partitioned into two disjoint sets: $\Delta_{\mathfrak{k}_{\mathbb{C}}}$ the set of compact roots and $\Delta_{\mathfrak{p}_{\mathbb{C}}}$ the set of imaginary noncompact roots. Let $\alpha_{p}$ be a noncompact imaginary simple root such that if $\beta=\sum_{i=1}^{l} c_{i} \alpha_{i}$ is a positive root, then $0 \leq c_{k} \leq 2$. Thus,

$$
\mathfrak{g}_{\mathbb{C}}=\mathfrak{g}_{\mathbb{C}}^{-2} \oplus \mathfrak{g}_{\mathbb{C}}^{-1} \oplus \mathfrak{g}_{\mathbb{C}}^{0} \oplus \mathfrak{g}_{\mathbb{C}}^{1} \oplus \mathfrak{g}_{\mathbb{C}}^{2}
$$

is a grading of $\mathfrak{g}_{\mathbb{C}}$, where $\mathfrak{g}_{\mathbb{C}}^{i}$ is the sum of the roots spaces for roots whose coefficient of $\alpha_{k}$ is $i$ in an expansion in terms of simple roots in $\Delta$. Define $l=\mathfrak{g}_{\mathbb{C}}^{0}$ and $u=\mathfrak{g}_{\mathbb{C}}^{1} \oplus \mathfrak{g}_{\mathbb{C}}^{2}$. Then $\mathfrak{q}=\mathfrak{l}+\mathfrak{u}$ is a maximal $\theta$-stable parabolic subalgebra of $\mathfrak{g}_{\mathbb{C}}$ and is called a Borel-de Siebenthal parabolic subalgebra. Furthermore, $\mathfrak{p}_{\mathbb{C}}=\mathfrak{g}_{\mathbb{C}}^{1} \oplus \mathfrak{g}_{\mathbb{C}}^{-1}$. Denote by $Q$ the connected subgroup of $G_{\mathbb{C}}$ with Lie algebra $\mathfrak{q}$. Then $\mathfrak{u} \cap \mathfrak{p}_{\mathbb{C}}=\mathfrak{g}_{\mathbb{C}}^{1}$ is a $Q \cap K_{\mathbb{C}}$-module under the adjoint action which we shall identify with its differential ad $: \mathfrak{q} \cap \mathfrak{k}_{\mathbb{C}} \rightarrow \mathfrak{u} \cap \mathfrak{p}_{\mathbb{C}}$

Theorem 2.3. Maintaining the above notation, $Q \cap K_{\mathbb{C}}$ has a unique open dense orbit in $\mathfrak{u} \cap \mathfrak{p}_{\mathbb{C}}$.

Proof. Observe that $\mathfrak{q} \cap \mathfrak{k}_{\mathbb{C}}=\mathfrak{g}_{\mathbb{C}}^{0} \oplus \mathfrak{g}_{\mathbb{C}}^{2}$ and that $\mathfrak{g}_{\mathbb{C}}^{2}$ acts trivially on $\mathfrak{g}_{\mathbb{C}}^{1}$. Therefore, the adjoint action of $\mathfrak{q} \cap \mathfrak{k}_{\mathbb{C}}$ on $\mathfrak{g}_{\mathbb{C}}^{1}$ is equivalent to that of $\mathfrak{g}_{\mathbb{C}}^{0}$ on $\mathfrak{g}_{\mathbb{C}}^{1}$. The theorem follows from Vinberg's theorem.

\section{RiCHARDSON ORBITS FOR REAL EXCEPTIONAL GROUPS}

Maintaining the above notation, we say that a nilpotent orbit $\mathcal{O}_{k}$ of $K_{\mathbb{C}}$ on $\mathfrak{p}_{\mathbb{C}}$ is a Richardson orbit if there exists a $\theta$-stable parabolic subalgebra $\mathfrak{q}$ of $\mathfrak{g}_{\mathbb{C}}$ with Levi decomposition $\mathfrak{q}=l \oplus \mathfrak{u}$ such that $\mathcal{O}_{k}$ is the unique dense orbit admitted by the saturation of $K_{\mathbb{C}}$ on $u \cap \mathfrak{p}_{\mathbb{C}}$. Following Peter Trapa we call $\mathcal{O}_{k}$ a $k$-form of the $G_{\mathbb{C}}$-orbit $\mathcal{O}$ if $\mathcal{O}_{k} \subseteq \mathcal{O} \cap \mathfrak{p}_{\mathbb{C}}$. In the case where $G_{\mathbb{C}}$ is a classical complex semisimple Lie group, Peter Trapa [8] proves the following theorem:

\footnotetext{
${ }^{1}$ Vogan systems define Vogan diagrams used to classify simple real Lie algebras; see [2] for more information.
} 
Theorem 3.1. Fix a special nilpotent orbit $\mathcal{O}$ of $G_{\mathbb{C}}$ on $\mathfrak{g}_{\mathbb{C}}$. Then there exists a real form $G$ such that some irreducible component of $\mathcal{O} \cap \mathfrak{p}_{\mathbb{C}}$ is a Richardson orbit of $K_{\mathbb{C}}$ on the nilpotent cone of $\mathfrak{p}_{\mathbb{C}}$.

The above theorem does not extend to exceptional groups. It fails for the minimal orbits of $E_{7}$ and $E_{8}$. Our search of the literature reveals that there is very little known about the vector space $\mathfrak{u} \cap \mathfrak{p}_{\mathbb{C}}$. Trapa's proof uses the fact that nilpotent orbits of $K_{\mathbb{C}}$ on $\mathfrak{p}_{\mathbb{C}}$ are parametrized by signed Young tableaux. We do not have such a parametrization for exceptional complex symmetric spaces. Instead we heavily use the parametrization given by Djoković [11, 12]. Our method is algorithmic, but does give complete information about each case. The results are given below.

The following proposition gives a necessary condition for a Richardson orbit and shows why the above question is indeed equivalent to Richardson's theorem in the complex case.

Proposition 3.2. Maintaining the above notation, let $\mathfrak{q}=\mathfrak{l} \oplus \mathfrak{u}$ be a $\theta$-stable parabolic subalgebra of $\mathfrak{g}_{\mathbb{C}}$ and let $e$ be a nilpotent element in $\mathfrak{p}_{\mathbb{C}}$ such that $\overline{\left(Q \cap K_{\mathbb{C}}\right) \cdot e}=$ $\mathfrak{u} \cap \mathfrak{p}_{\mathbb{C}}$. Then $\overline{K_{\mathbb{C}} \cdot e} \cap\left(\mathfrak{u} \cap \mathfrak{p}_{\mathbb{C}}\right)$ is open and dense in $u \cap \mathfrak{p}_{\mathbb{C}}$.

Proof. The fact that $K_{\mathbb{C}}=K \times Q \cap K_{\mathbb{C}}$ implies that $K_{\mathbb{C}} \cdot e=K \times\left(Q \cap K_{\mathbb{C}}\right) \cdot e$. Since $\mathfrak{u} \cap \mathfrak{p}_{\mathbb{C}}$ is a $Q \cap K_{\mathbb{C}}$-module we must have

$$
K_{\mathbb{C}} \cdot e \cap\left(\mathfrak{u} \cap \mathfrak{p}_{\mathbb{C}}\right)=K \cdot e \cap\left(\mathfrak{u} \cap \mathfrak{p}_{\mathbb{C}}\right) .
$$

Suppose that there exists $e^{\prime} \in \mathfrak{u} \cap \mathfrak{p}_{\mathbb{C}}$ such that $e^{\prime} \neq e$ and $K \cdot e^{\prime} \cap \mathfrak{u} \cap \mathfrak{p}_{\mathbb{C}}$ is open in $\mathfrak{u} \cap \mathfrak{p}_{\mathbb{C}}$. Then we can find a sequence $\left\{q_{n}\right\}$ in $Q$ such that $q_{n}(e) \rightarrow e^{\prime}$ for $\overline{\left(Q \cap K_{\mathbb{C}}\right) \cdot e}=\mathfrak{u} \cap \mathfrak{p}_{\mathbb{C}}$. Hence $K \cdot q_{n}(e) \rightarrow K \cdot e^{\prime}$ and $\overline{K_{\mathbb{C}} \cdot e} \cap\left(\mathfrak{u} \cap \mathfrak{p}_{\mathbb{C}}\right)$ is open and dense in $u \cap \mathfrak{p}_{\mathbb{C}}$.

From a geometrical point of view Richardson orbits arise as dense $K_{\mathbb{c}}$-orbits in the moment map image of conormal bundles to certain orbits $\mathcal{O}_{\mathfrak{q}}$ defined by $\mathfrak{q}$. Let $\mathcal{A}_{\mathfrak{q}}$ be the irreducible Harish-Chandra module of trivial infinitesimal character attached to the trivial local system on $\mathcal{O}_{\mathfrak{q}}$ by the Beilinson-Bernstein equivalence. Then, in the context of representation theory, we may think of the Richardson orbits as $K_{\mathbb{C}}$-nilpotent orbits of in $\mathfrak{p}_{\mathbb{C}}$ which are dense in the associated varieties of modules of the form $\mathcal{A}_{\mathfrak{q}}$. See [8] for more details. Using the above theorem Trapa was able to compute explicitly the annihilator of any module of the form $\mathcal{A}_{\mathfrak{q}}$ for the classical groups. This allowed him to give new examples of simple highest weight modules with irreducible associate varieties.

Definition 3.3. Let $\mathfrak{q}=\mathfrak{q} \cap \mathfrak{k}_{\mathbb{C}} \oplus \mathfrak{q} \cap \mathfrak{p}_{\mathbb{C}}$ be a $\theta$ stable parabolic subalgebra of $\mathfrak{g}_{\mathbb{C}}$ with Levi decomposition $\mathfrak{q}=\mathfrak{l} \oplus \mathfrak{u}$. Let $e$ be a nilpotent element of $u \cap \mathfrak{p}_{\mathbb{C}}$. We say that $\mathfrak{q}$ is a polarization of $\mathfrak{g}_{\mathrm{C}}$ at $e$ if

$$
2 \operatorname{dim} \mathfrak{q}=\operatorname{dim}_{\mathbb{C}}^{e}+\operatorname{dim}_{\mathbb{C}} \quad \text { and } \quad B(e,[\mathfrak{q}, \mathfrak{q}])=0,
$$

where $\mathfrak{g}_{\mathbb{C}}^{e}$ is the centralizer of $e$ in $\mathfrak{g}_{\mathbb{C}}$ and $B$ is the Killing form of $\mathfrak{g}_{\mathbb{C}}$.

The next proposition could be seen as a version of Richardson's theorem for complex symmetric spaces.

Proposition 3.4 (P. Tauvel). Maintaining the above notation, suppose that there exists $z$ in $\mathfrak{p}_{\mathbb{C}}$ such that $\mathfrak{q}$ is a polarization of of $\mathfrak{g}_{\mathbb{C}}$ at $z$. Then

(i) There exists a unique $K_{\mathbb{C}}$-nilpotent orbit $\mathcal{O}_{k}$ in $\mathfrak{p}_{\mathbb{C}}$ such that $\mathcal{S}=\mathfrak{u} \cap \mathfrak{p}_{\mathbb{C}} \cap \mathcal{O}_{k}$ is an open and dense in $\mathfrak{u} \cap \mathfrak{p}_{\mathbb{C}}$. 
(ii) $\mathcal{S}$ is a $Q \cap K_{\mathbb{C}}$-orbit.

(iii) If $x \in \mathcal{S}$ then $\left[x, \mathfrak{k}_{\mathbb{C}} \cap \mathfrak{q}\right]=\mathfrak{u} \cap \mathfrak{p}_{\mathbb{C}},\left[x, \mathfrak{p}_{\mathbb{C}} \cap \mathfrak{q}\right]=\mathfrak{u} \cap \mathfrak{k}_{\mathbb{C}}$ an $\mathfrak{q}$ is a polarization of $\mathfrak{g}_{\mathbb{C}}$ at $x$.

Proof. See [7, proposition 4.6].

Definition 3.5. A nilpotent $e \in \mathfrak{p}_{\mathbb{C}}$ is polarizable if there exists a $\theta$-stable parabolic $\mathfrak{q}$ such that $\mathfrak{q}$ is a polarization of $\mathfrak{g}_{\mathbb{C}}$ at $e$. A nilpotent orbit of $K_{\mathbb{C}}$ on $\mathfrak{p}_{\mathbb{C}}$ is polarizable if at least one of its representatives is polarizable. A nilpotent orbit $\mathcal{O}$ of $G_{\mathbb{C}}$ on $\mathfrak{g}_{\mathbb{C}}$ is polarizable if at least one of its $k$-forms is polarizable.

There exist nonpolarizable nilpotent elements. An example of such elements is given in [7, p. 644] for $\mathfrak{g}_{\mathbb{C}}=A_{1}$.

Our goal is to analyze to what extent Peter Trapa's theorem fails for exceptional groups. From now on all statements are concerned with exceptional groups.

Consider the real forms $G, F I, E I I, E V, E V I I I$ of $G_{2}, F_{4}, E_{6}, E_{7}$, and $E_{8}$, respectively. Then each even nilpotent orbit $\mathcal{O}$ of $G_{\mathbb{C}}$ has a $k$-form $\mathcal{O}_{k}$. The JacobsonMorozov parabolic subalgebra $\mathfrak{q}$ associated with any representative $e$ of $\mathcal{O}_{k}$ is a polarization $\mathfrak{g}_{\mathbb{C}}$ at $e$.

In order to decide whether a given orbit is polarizable, one can compute the list of all representatives of the $K_{\mathbb{C}}$-conjugacy classes of theta stable parabolic subalgebras containing a representative of the orbit. Once such a list is available, then one could look at a parabolic subalgebra with the appropriate dimension. The algorithm used to find the theta-stable parabolic subalgebras and some implementation details in the software LiE [10] are given in [4].

The tables below contains the following information:

1. The Bala-Carter label for the complex $\mathcal{O}$.

2. A representative $e$ of $\mathcal{O}_{k}$.

3. The real form of $\mathfrak{g}_{\mathbb{C}}$ relative to the Cartan involution $\theta$.

4. The $\theta$-stable parabolic $\mathfrak{q}=l+\mathfrak{u}$.

5. When $\mathcal{O}$ is polarizable then the dimension of $\mathfrak{q}$ and that of $\mathfrak{g}_{\mathbb{C}}^{e}$ are given. $G_{2}$.

If $\mathfrak{g}_{\mathbb{C}}=G_{2}$, then there are no noneven special orbits. Hence Trapa's theorem applies. Moreover none of the two noneven nilpotent orbits is polarizable. An easy computation shows that the dimension of any $\theta$-stable parabolic subalgebra is either 8 or 9 , while the two orbits have dimensions 6 and 8 , respectively.

$F_{4}$.

Proposition 3.6. Let $\mathfrak{g}_{\mathbb{C}}=F_{4}$ and fix a special nilpotent orbit $\mathcal{O}$ of $G_{\mathbb{C}}$ on $\mathfrak{g}_{\mathbb{C}}$. Then there exists a real form $G$ such that some irreducible component of $\mathcal{O} \cap \mathfrak{p}_{\mathbb{C}}$ is a Richardson orbit of $K_{\mathbb{C}}$ on the nilpotent cone of $\mathfrak{p}_{\mathbb{C}}$.

Proof. From previous considerations, it is enough to establish the proposition for special noneven orbits. There are exactly three such orbits, $\tilde{A}_{1}, A_{1}+\tilde{A}_{1}$ and $C_{3}$.

We order the roots of $F_{4}$ as in the table below, and we use the Bourbaki system of simple roots $\Delta=\left\{\alpha_{1}, \alpha_{2}, \alpha_{3}, \alpha_{4}\right\}$. The Cartan involution $\theta$ with +1 -eigenspace $\mathfrak{k}$ and -1-eigenspace $\mathfrak{p}$ depends on the real forms. If $\mathfrak{g}_{\mathbb{R}}=F I$, then $\mathfrak{k}_{\mathbb{C}}=\mathfrak{s p}_{3}(\mathbb{C}) \oplus$ $\mathfrak{s l}_{2}(\mathbb{C})$, and the vector space $\mathfrak{p}_{\mathbb{C}}$ is the complex span of nonzero root vectors $X_{\beta}$, where $\beta=c_{1} \alpha_{1}+c_{2} \alpha_{2}+c_{3} \alpha_{3}+c_{4} \alpha_{4}$ with $c_{1}= \pm 1$. If $\mathfrak{g}_{\mathbb{R}}=F I I$, then $\mathfrak{k}_{\mathbb{C}}=\mathfrak{s o}_{9}(\mathbb{C})$, and the vector space $\mathfrak{p}_{\mathbb{C}}$ is the complex span of nonzero root vectors $X_{\beta}$, where $\beta=c_{1} \alpha_{1}+c_{2} \alpha_{2}+c_{3} \alpha_{3}+c_{4} \alpha_{4}$ with $c_{4}= \pm 1$. 


\begin{tabular}{lrr}
\hline & \multicolumn{2}{c}{ Positive roots of $F_{4}$} \\
\hline 1. $[1,0,0,0]$ & $9 .[0,1,2,0]$ & $17 .[1,2,2,1]$ \\
2. $[0,1,0,0]$ & 10. $[0,1,1,1]$ & $18 .[1,1,2,2]$ \\
3. $[0,0,1,0]$ & $11 .[1,1,2,0]$ & $19 .[1,2,3,1]$ \\
4. $[0,0,0,1]$ & 12. $[1,1,1,1]$ & $20 .[1,2,2,2]$ \\
5. $[1,1,0,0]$ & 13. $[0,1,2,1]$ & $21 .[1,2,3,2]$ \\
6. $[0,1,1,0]$ & 14. $[1,2,2,0]$ & $22 .[1,2,4,2]$ \\
7. $[0,0,1,1]$ & 15. $[1,1,2,1]$ & $23 .[1,3,4,2]$ \\
8. $[1,1,1,0]$ & 16. $[0,1,2,2]$ & $24 .[2,3,4,2]$ \\
\hline \hline
\end{tabular}

In the preceding table each vector indexed by $i$ represents the coefficients of the $i$ th positive root in the Bourbaki base $\Delta$. We use $X_{i}$ or $X_{-i}$ to denote a nonzero root vector in the root spaces of $i$ and $-i$, respectively.

The orbit labeled $C_{3}$ of dimension 42 is polarizable as indicated below.

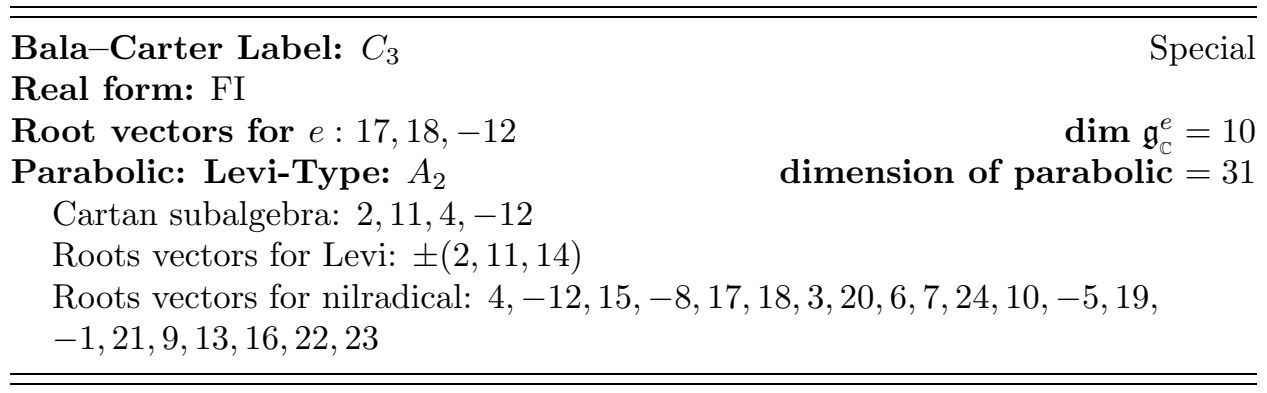

The two tables that follow the proof contain the representatives of the $K_{\mathrm{C}}$-conjugacy classes of all theta parabolic subalgebras relative to both real forms of $F_{4}$. From the first table, it is easy to check that all $\theta$-stable parabolic subalgebra of $F_{4}$ relative to $F I$ except $\mathfrak{q}_{12}=l_{12} \oplus \mathfrak{u}_{12}$ and $\mathfrak{q}_{24}=l_{24} \oplus \mathfrak{u}_{24}$ labeled 12 and 24 contain a nilpotent of the form $\left(X_{\alpha}+X_{\beta}\right)$, where the roots $\alpha$ and $\beta$ are in $\mathfrak{p}_{\mathbb{C}}$, and generate an algebra of type $\tilde{A}_{2}$ or $A_{2}$, which represents $K_{\mathbb{C}}$-orbits of dimension 15 . But $\mathfrak{u}_{24} \cap \mathfrak{p}_{\mathbb{C}}$ contains the following nilpotent $X_{1}+X_{14}+X_{21}$ representing the orbit $A_{1}+A_{1}+\tilde{A}_{1}$ which is a $k$-form of $A_{2}$. Moreover the product of any two roots in $\mathfrak{u}_{12} \cap \mathfrak{p}_{\mathbb{C}}$ is nonnegative, that is, all the root vectors commute. Hence the nilpotent orbit labeled $A_{1}+\tilde{A}_{1}$ represented by $X_{22}+X_{12}$ in $\mathfrak{u}_{12} \cap \mathfrak{p}_{\mathbb{C}}$ of dimension 14 must intersect $\mathfrak{u}_{12} \cap \mathfrak{p}_{\mathbb{C}}$ in an open dense set. Therefore, if we fixed the orbit $A_{1}+\tilde{A}_{1}$, then $q_{12}$ will satisfy the proposition. The above discussion is summarized below.

\begin{tabular}{l}
\hline \hline Bala-Carter Label: $A_{1}+\tilde{A}_{1}$ \\
Real form: FI \\
Root vectors for $e: 22,12$ \\
Parabolic: Levi-Type: $B_{3}$ \\
$\quad$ Cartan subalgebra: $1,2,3,4$ \\
$\quad$ Roots vectors for Levi: $\pm(1,2,3,5,6,8,9,11,14)$ \\
Roots vectors for nilradical: $4,7,10,12,13,15,16,17,18,19,20,21,22,23,24$ \\
\hline
\end{tabular}


Clearly, in order to resolve the orbit of type $\tilde{A}_{1}$ we need to consider the other real form $F I I$. From the second table below it is easy to check that all $\theta$-stable parabolic subalgebra of $F_{4}$ relative to $F I I$, except $\mathfrak{q}_{15}=l_{15} \oplus \mathfrak{u}_{15}$ and $\mathfrak{q}_{24}=l_{24} \oplus \mathfrak{u}_{24} \cap \mathfrak{p}_{\mathbb{C}}$, labeled 15 and 24, contain a nilpotent of the form $\left(X_{\alpha}+X_{\beta}\right)$, where the roots $\alpha$ and $\beta$ are in $\mathfrak{p}$ and generate an algebra of type $\tilde{A}_{2}$ which represents the $K$-orbit of dimension 15 . Since there are only two nilpotent classes, the other being of type $\tilde{A}_{1}, q_{24}$ satisfies the proposition.

\begin{tabular}{l} 
Bala-Carter Label: $\tilde{A}_{1}$ \\
Real form: FII \\
Root vectors for $e:-4$ \\
Parabolic: Levi-Type: $B_{3}$ \\
$\quad$ Cartan subalgebra: $1,2,7,-4$ \\
$\quad$ Roots vectors for Levi: $\pm(1,2,7,5,10,12,16,18,20)$ \\
$\quad$ Roots vectors for nilradical: $-4,3,6,8,13,15,9,17,11,21,14,19,22,23,24$ \\
\hline
\end{tabular}

This concludes the proof.

For the benefit of the reader we describe the algorithm in [4] for the case of $F_{4}$. The reader should realize that the same algorithm works for all real Lie groups of inner type. Some changes were needed for EIV. However since there are only two nilpotent orbits in $E I V$, our result for that specific group can be easily verified. The next two tables were generated as follows:

1. For each real form we compute the $K_{\mathbb{C}}$-conjugacy classes of systems of simple roots. Starting with a Vogan diagram we obtain the other nonconjugate diagrams by reflecting along noncompact imaginary roots. Observe that the number of such classes is $\frac{W\left(G_{\mathbb{C}}\right)}{W\left(K_{\mathbb{C}}\right)}$, where $W\left(G_{\mathbb{C}}\right)$ and $W\left(K_{\mathbb{C}}\right)$ are the Weyl groups of $G_{\mathbb{C}}$ and $K_{\mathbb{C}}$, respectively. Hence there are 12 classes for $F I$ and three for $F I I$. For information about Vogan diagrams consult [2].

2. For each class of simple roots $\Delta$, we build standard parabolic subalgebras by using the subsets of $\Delta$. Observe that because the ranks of $G$ and $K$ are equal, all such parabolic subalgebras must be $\theta$-stable. We also eliminate duplicates in the process.

The computation was implemented in the software LiE. It is easy to see that the final lists must contain a representative of each $K$-conjugacy class of $\theta$-stable parabolic subalgebras relative to each real form.

To find the pairs of roots generating a nilpotent of type $\tilde{A}_{2}$ or $A_{2}$, we traverse the nilradical of each parabolic subalgebra in our lists and look for appropriate pairs of short and long roots. This is easily done in LiE using built-in functions. Information on $\mathrm{LiE}$ is found in [10].

$\theta$-stable parabolic subalgebras relative to $F I$

\begin{tabular}{ll}
\hline \hline 1. & $\mathfrak{h}=(1,2,3,4) \oplus \mathfrak{u}=1,2,3,4,5,6,7,8,9,10,11,12,13,14,15,16,17,18,19$, \\
& $20,21,22,23,24$ \\
\hline 2. & $\mathfrak{l}=\mathfrak{h} \oplus \pm(1) \oplus \mathfrak{u}=2,3,4,5,6,7,8,9,10,11,12,13,14,15,16,17,18,19$, \\
& $20,21,22,23,24$
\end{tabular}


3. $\mathfrak{l}=\mathfrak{l} \oplus \pm(2) \oplus \mathfrak{u}=1,3,4,5,6,7,8,9,10,11,12,13,14,15,16,17,18,19,20,21$, $22,23,24$

4. $\quad \mathfrak{l}=\mathfrak{h} \oplus \pm(3) \oplus \mathfrak{u}=1,2,4,5,6,7,8,9,10,11,12,13,14,15,16,17,18,19,20,21$, $22,23,24$

5. $\mathfrak{l}=\mathfrak{h} \oplus \pm(4) \oplus \mathfrak{u}=1,2,3,5,6,7,8,9,10,11,12,13,14,15,16,17,18,19,20,21$, $22,23,24$

6. $\quad \mathfrak{l}=\mathfrak{h} \oplus \pm(1,2,5) \oplus \mathfrak{u}=3,4,6,7,8,9,10,11,12,13,14,15,16,17,18,19,20,21$, $22,23,24$

7. $\mathfrak{l}=\mathfrak{h} \oplus \pm(1,3) \oplus \mathfrak{u}=2,4,5,6,7,8,9,10,11,12,13,14,15,16,17,18,19,20,21$, $22,23,24$

8. $\quad \mathfrak{l}=\mathfrak{h} \oplus \pm(1,4) \oplus \mathfrak{u}=2,3,5,6,7,8,9,10,11,12,13,14,15,16,17,18,19,20,21$, $22,23,24$

$9 . \quad \mathfrak{l}=\mathfrak{h} \oplus \pm(2,3,6,9) \oplus \mathfrak{u}=1,4,5,7,8,10,11,12,13,14,15,16,17,18,19,20,21$, $22,23,24$

$10 . \quad \mathfrak{l}=\mathfrak{h} \oplus \pm(2,4) \oplus \mathfrak{u}=1,3,5,6,7,8,9,10,11,12,13,14,15,16,17,18,19,20,21$, $22,23,24$

11. $\mathfrak{l}=\mathfrak{h} \oplus \pm(3,4,7) \oplus \mathfrak{u}=1,2,5,6,8,9,10,11,12,13,14,15,16,17,18,19,20,21$, $22,23,24$

$12 . \quad \mathfrak{l}=\mathfrak{h} \oplus \pm(1,2,3,5,6,8,9,11,14) \oplus \mathfrak{u}=4,7,10,12,13,15,16,17,18,19,20,21$, $22,23,24$

13. $\mathfrak{l}=\mathfrak{h} \oplus \pm(1,2,5,4) \oplus \mathfrak{u}=3,6,7,8,9,10,11,12,13,14,15,16,17,18,19,20,21$, $22,23,24$

$14 . \quad \mathfrak{l}=\mathfrak{h} \oplus \pm(4,3,7,1) \oplus \mathfrak{u}=2,5,6,8,9,10,11,12,13,14,15,16,17,18,19,20,21$, $22,23,24$

$15 . \mathfrak{l}=\mathfrak{h} \oplus \pm(4,3,2,7,6,10,9,13,16) \oplus \mathfrak{u}=1,5,8,11,12,14,15,17,18,19,20,21$, $22,23,24$

16. $\mathfrak{l}=F_{4} \oplus \mathfrak{u}=0$

17. $\mathfrak{h}=(-1,5,3,4) \oplus \mathfrak{u}=-1,5,3,4,2,8,7,6,11,12,9,10,15,14,13,18,17,16$, $19,20,21,22,24,23$

18. $\mathfrak{l}=\mathfrak{h} \oplus \pm(5) \oplus \mathfrak{u}=-1,3,4,2,8,7,6,11,12,9,10,15,14,13,18,17,16,19,20$, $21,22,24,23$

$19 . \quad \mathfrak{l}=\mathfrak{h} \oplus \pm(3) \oplus \mathfrak{u}=-1,5,4,2,8,7,6,11,12,9,10,15,14,13,18,17,16,19,20$, $21,22,24,23$

$20 . \quad \mathfrak{l}=\mathfrak{h} \oplus \pm(4) \oplus \mathfrak{u}=-1,5,3,2,8,7,6,11,12,9,10,15,14,13,18,17,16,19,20$, $21,22,24,23$

$21 . \quad \mathfrak{l}=\mathfrak{h} \oplus \pm(5,3,8,11) \oplus \mathfrak{u}=-1,4,2,7,6,12,9,10,15,14,13,18,17,16,19,20$, $21,22,24,23$

$22 . \quad \mathfrak{l}=\mathfrak{h} \oplus \pm(5,4) \oplus \mathfrak{u}=-1,3,2,8,7,6,11,12,9,10,15,14,13,18,17,16,19,20$, $21,22,24,23$

$23 . \quad \mathfrak{l}=\mathfrak{h} \oplus \pm(3,4,7) \oplus \mathfrak{u}=-1,5,2,8,6,11,12,9,10,15,14,13,18,17,16,19,20$, $21,22,24,23$

$24 . \quad \mathfrak{l}=\mathfrak{h} \oplus \pm(4,3,5,7,8,12,11,15,18) \oplus \mathfrak{u}=-1,2,6,9,10,14,13,17,16,19,20$, $21,22,24,23$

$25 . \quad \mathfrak{h}=(2,-5,8,4) \oplus \mathfrak{u}=2,-5,8,4,-1,3,12,6,11,7,14,10,15,9,17,18,13,20$, $19,16,21,24,22,23$

$26 . \quad \mathfrak{l}=\mathfrak{h} \oplus \pm(2) \oplus \mathfrak{u}=-5,8,4,-1,3,12,6,11,7,14,10,15,9,17,18,13,20,19$, $16,21,24,22,23$

$\frac{16}{27 .} \quad \mathfrak{l}=\mathfrak{h} \oplus \pm(8) \oplus \mathfrak{u}=2,-5,4,-1,3,12,6,11,7,14,10,15,9,17,18,13,20,19$, $16,21,24,22,23$

$28 . \quad \mathfrak{l}=\mathfrak{h} \oplus \pm(4) \oplus \mathfrak{u}=2,-5,8,-1,3,12,6,11,7,14,10,15,9,17,18,13,20,19$, $16,21,24,22,23$ 
$29 . \quad \mathfrak{l}=\mathfrak{h} \oplus \pm(2,8) \oplus \mathfrak{u}=-5,4,-1,3,12,6,11,7,14,10,15,9,17,18,13,20,19$, $16,21,24,22,23$

$30 . \quad \mathfrak{l}=\mathfrak{h} \oplus \pm(2,4) \oplus \mathfrak{u}=-5,8,-1,3,12,6,11,7,14,10,15,9,17,18,13,20,19$, $16,21,24,22,23$

31. $\mathfrak{l}=\mathfrak{h} \oplus \pm(8,4,12) \oplus \mathfrak{u}=2,-5,-1,3,6,11,7,14,10,15,9,17,18,13,20,19$, $16,21,24,22,23$

$32 . \quad \mathfrak{l}=\mathfrak{h} \oplus \pm(4,6,12,2) \oplus \mathfrak{u}=-5,-1,3,6,11,7,14,10,15,9,17,18,13,20,19$, $16,21,24,22,23$

33. $\mathfrak{h}=(2,11,-8,12) \oplus \mathfrak{u}=2,11,-8,12,14,3,4,6,-5,15,-1,17,7,9,10,18$, $19,20,13,24,21,16,22,23$

$\frac{1}{34 . \quad \mathfrak{l}=\mathfrak{h} \oplus \pm(2) \oplus \mathfrak{u}=11,-8,12,14,3,4,6,-5,15,-1,17,7,9,10,18,19,20,13,}$ $24,21,16,22,23$

$35 . \quad \mathfrak{l}=\mathfrak{h} \oplus \pm(11) \oplus \mathfrak{u}=2,-8,12,14,3,4,6,-5,15,-1,17,7,9,10,18,19,20,13$, $24,21,16,22,23$

$36 . \quad \mathfrak{l}=\mathfrak{h} \oplus \pm(12) \oplus \mathfrak{u}=2,11,-8,14,3,4,6,-5,15,-1,17,7,9,10,18,19,20,13$, $24,21,16,22,23$

$37 . \quad \mathfrak{l}=\mathfrak{h} \oplus \pm(2,11,14) \oplus \mathfrak{u}=-8,12,3,4,6,-5,15,-1,17,7,9,10,18,19,20,13$, $24,21,16,22,23$

$38 . \quad \mathfrak{l}=\mathfrak{h} \oplus \pm(2,12) \oplus \mathfrak{u}=11,-8,14,3,4,6,-5,15,-1,17,7,9,10,18,19,20,13$, $24,21,16,22,23$

$39 . \quad \mathfrak{l}=\mathfrak{h} \oplus \pm(11,12) \oplus \mathfrak{u}=2,-8,14,3,4,6,-5,15,-1,17,7,9,10,18,19,20,13$, $24,21,16,22,23$

$40 . \quad \mathfrak{l}=\mathfrak{h} \oplus \pm(2,11,14,12) \oplus \mathfrak{u}=-8,3,4,6,-5,15,-1,17,7,9,10,18,19,20,13$, $24,21,16,22,23$

41. $\mathfrak{h}=(14,-11,3,12) \oplus \mathfrak{u}=14,-11,3,12,2,-8,15,6,-5,4,9,17,7,-1,19$, $18,10,24,13,20,21,22,16,23$

$42 . \quad \mathfrak{l}=\mathfrak{h} \oplus \pm(14) \oplus \mathfrak{u}=-11,3,12,2,-8,15,6,-5,4,9,17,7,-1,19,18,10,24$, $13,20,21,22,16,23$

43. $\mathfrak{l}=\mathfrak{h} \oplus \pm(3) \oplus \mathfrak{u}=14,-11,12,2,-8,15,6,-5,4,9,17,7,-1,19,18,10,24$, $13,20,21,22,16,23$

44. $\quad \mathfrak{l}=\mathfrak{h} \oplus \pm(12) \oplus \mathfrak{u}=14,-11,3,2,-8,15,6,-5,4,9,17,7,-1,19,18,10,24$, $13,20,21,22,16,23$

$45 . \mathfrak{l}=\mathfrak{h} \oplus \pm(14,3) \oplus \mathfrak{u}=-11,12,2,-8,15,6,-5,4,9,17,7,-1,19,18,10,24$, $13,20,21,22,16,23$

46. $\quad \mathfrak{l}=\mathfrak{h} \oplus \pm(14,12) \oplus \mathfrak{u}=-11,3,2,-8,15,6,-5,4,9,17,7,-1,19,18,10,24$, $13,20,21,22,16,23$

47. $\mathfrak{l}=\mathfrak{h} \oplus \pm(3,12,15) \oplus \mathfrak{u}=14,-11,2,-8,6,-5,4,9,17,7,-1,19,18,10,24$, $13,20,21,22,16,23$

48. $\mathfrak{l}=\mathfrak{h} \oplus \pm(3,12,15,14) \oplus \mathfrak{u}=-11,2,-8,6,-5,4,9,17,7,-1,19,18,10,24$, $13,20,21,22,16,23$

$49 . \quad \mathfrak{h}=(2,11,4,-12) \oplus \mathfrak{u}=2,11,4,-12,14,15,-8,17,, 18,3,20,6,7,24,10$, $-5,19,-1,21,9,13,16,22,23$

$50 . \quad \mathfrak{l}=\mathfrak{h} \oplus \pm(2) \oplus \mathfrak{u}=11,4,-12,14,15,-8,17,18,3,20,6,7,24,10,-5,19$, $-1,21,9,13,16,22,23$

$51 . \quad l=\mathfrak{h} \oplus \pm(11) \oplus \mathfrak{u}=2,4,-12,14,15,-8,17,18,3,20,6,7,24,10,-5,19$, $-1,21,9,13,16,22,23$

$52 . \quad \mathfrak{l}=\mathfrak{h} \oplus \pm(4) \oplus \mathfrak{u}=2,11,-12,14,15,-8,17,18,3,20,6,7,24,10,-5,19$, $-1,21,9,13,16,22,23$

$53 . \quad \mathfrak{l}=\mathfrak{h} \oplus \pm(2,11,14) \oplus \mathfrak{u}=4,-12,15,-8,17,18,3,20,6,7,24,10,-5,19$, $-1,21,9,13,16,22,23$

$54 . \mathfrak{l}=\mathfrak{h} \oplus \pm(2,4) \oplus \mathfrak{u}=11,-12,14,15,-8,17,18,3,20,6,7,24,10,-5,19$, $-1,21,9,13,16,22,23$ 
$55 . \quad \mathfrak{l}=\mathfrak{h} \oplus \pm(11,4,15,18) \oplus \mathfrak{u}=2,-12,14,-8,17,3,20,6,7,24,10,-5,19$, $-1,21,9,13,16,22,23$

56. $\mathfrak{l}=\mathfrak{h} \oplus \pm(2,11,4,14,15,17,18,20,24) \oplus \mathfrak{u}=-12,-8,3,6,7,10,-5,19$, $-1,21,9,13,16,22,23$

$57 . \mathfrak{h}=(-14,2,3,12) \oplus \mathfrak{u}=-14,2,3,12,-11,6,15,-8,9,17,-5,4,19,-1$, $7,24,10,18,13,20,21,22,23,16$ $58 . \quad \mathfrak{l}=\mathfrak{h} \oplus \pm(2) \oplus \mathfrak{u}=-14,3,12,-11,6,15,-8,9,17,-5,4,19,-1$, $7,24,10,18,13,20,21,22,23,16$

59. $\mathfrak{l}=\mathfrak{h} \oplus \pm(3) \oplus \mathfrak{u}=-14,2,12,-11,6,15,-8,9,17,-5,4,19,-1,7,24,10$, $18,13,20,21,22,23,16$ $60 . \mathfrak{l}=\mathfrak{h} \oplus \pm(12) \oplus \mathfrak{u}=-14,2,3,-11,6,15,-8,9,17,-5,4,19,-1,7,24,10$, $18,13,20,21,22,23,16$

61. $\mathfrak{l}=\mathfrak{h} \oplus \pm(2,3,6,9) \oplus \mathfrak{u}=-14,12,-11,15,-8,17,-5,4,19,-1,7,24,10$, $18,13,20,21,22,23,16$ $62 . \mathfrak{l}=\mathfrak{h} \oplus \pm(2,12) \oplus \mathfrak{u}=-14,3,-11,6,15,-8,9,17,-5,4,19,-1,7,24,10$, $18,13,20,21,22,23,16$

63. $l=\mathfrak{h} \oplus \pm(3,12,15) \oplus \mathfrak{u}=-14,2,-11,6,-8,9,17,-5,4,19,-1,7,24,10$, $18,13,20,21,22,23,16$ $64 . \mathfrak{l}=\mathfrak{h} \oplus \pm(12,3,2,15,6,17,9,19,24) \oplus \mathfrak{u}=-14,-11,-8,-5,4,-1,7,10$, $18,13,20,21,22,23,16$

$65 . \mathfrak{h}=(14,-11,15,-12) \oplus \mathfrak{u}=14,-11,15,-12,2,4,3,17,18,-8,24,6,7,20$, $19,-5,10,9,21,-1,13,22,16,23$ $66 . \mathfrak{l}=\mathfrak{h} \oplus \pm(14) \oplus \mathfrak{u}=-1115,-12,2,4,3,17,18,-8,24,6,7,20,19,-5,10$ $9,21,-1,13,22,16,23$

67. $\mathfrak{l}=\mathfrak{h} \oplus \pm(15) \oplus \mathfrak{u}=14,-11,-12,2,4,3,17,18,-8,24,6,7,20,19,-5,10$, $9,21,-1,13,22,16,23$ 68. $\mathfrak{l}=\mathfrak{h} \oplus \pm(14,15) \oplus \mathfrak{u}=-11,-12,2,4,3,17,18,-8,24,6,7,20,19,-5,10$ $9,21,-1,13,22,16,23$

$69 . \mathfrak{h}=(-14,2,15,-12) \oplus \mathfrak{u}=-14,2,15,-12,-11,17,3,4,24,6,18,-8,19$, $20,7,9,10,-5,21,-1,13,22,23,16$ $70 . \mathfrak{l}=\mathfrak{h} \oplus \pm(2) \oplus \mathfrak{u}=-14,15,-12,-11,17,3,4,24,6,18,-8,19,20,7,9,10$, $-5,21,-1,13,22,23,16$

71. $\mathfrak{l}=\mathfrak{h} \oplus \pm(15) \oplus \mathfrak{u}=-14,2,-12,-11,17,3,4,24,6,18,-8,19,20,7,9,10$, $-5,21,-1,13,22,23,16$

72. $\mathfrak{l}=\mathfrak{h} \oplus \pm(2,15,17,24) \oplus \mathfrak{u}=-14,-12,-11,3,4,6,18,-8,19,20,7,9,10$, $-5,21,-1,13,22,23,16$

73. $\mathfrak{h}=(14,18,-15,3) \oplus \mathfrak{u}=14,18,-15,3,24,4,-12,17,-11,7,2,19,-8,20$, $6,-5,21,9,10,22,13,-1,16,23$

74. $\mathfrak{l}=\mathfrak{h} \oplus \pm(14) \oplus \mathfrak{u}=18,-15,3,24,4,-12,17,-11,7,2,19,-8,20,6,-5,21$, $9,10,22,13,-1,16,23$

$75 . \mathfrak{l}=\mathfrak{h} \oplus \pm(18) \oplus \mathfrak{u}=14,-15,3,24,4,-12,17,-11,7,2,19,-8,20,6,-5,21$, $9,10,22,13,-1,16,23$

76. $\mathfrak{l}=\mathfrak{h} \oplus \pm(3) \oplus \mathfrak{u}=14,18,-15,24,4,-12,17,-11,7,2,19,-8,20,6,-5,21$, $9,10,22,13,-1,16,23$

77. $l=\mathfrak{h} \oplus \pm(14,18,24) \oplus \mathfrak{u}=-15,3,4,-12,17,-11,7,2,19,-8,20,6,-5,21$, $9,10,22,13,-1,16,23$

78. $\mathfrak{l}=\mathfrak{h} \oplus \pm(14,3) \oplus \mathfrak{u}=18,-15,24,4,-12,17,-11,7,2,19,-8,20,6,-5,21$, $9,10,22,13,-1,16,23$

$79 . \quad \mathfrak{l}=\mathfrak{h} \oplus \pm(18,3) \oplus \mathfrak{u}=14,-15,24,4,-12,17,-11,7,2,19,-8,20,6,-5,21$, $9,10,22,13,-1,16,23$

80. $\mathfrak{l}=\mathfrak{h} \oplus \pm(14,18,24,3) \oplus \mathfrak{u}=-15,4,-12,17,-11,7,2,19,-8,20,6,-5,21$, $9,10,22,13,-1,16,23$ 


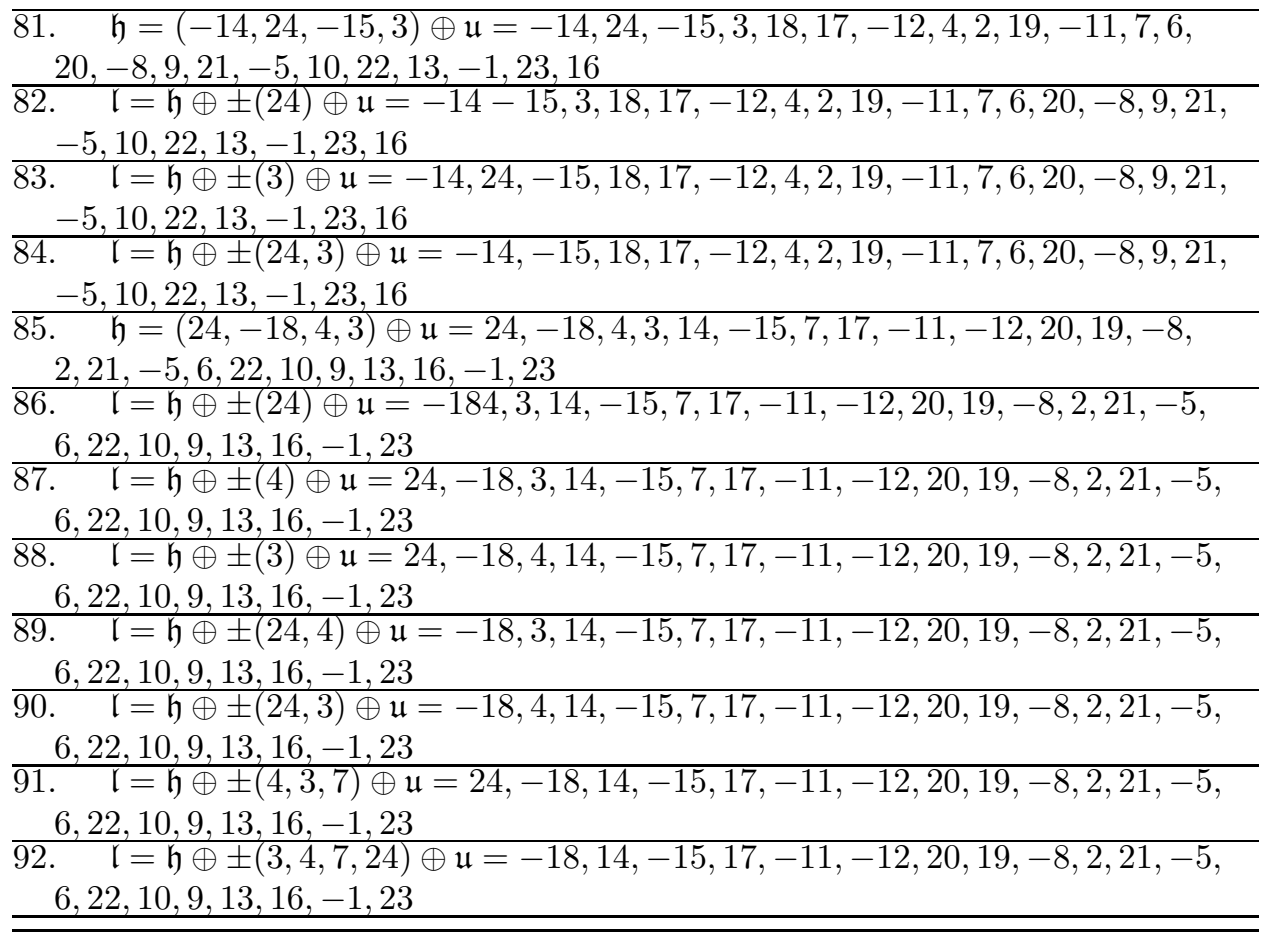

\section{$\theta$-stable parabolic subalgebras relative to $F I I$}

The first 16 parabolic subalgebras are listed in the previous table in the same order.

$\overline{17 . \quad \mathfrak{h}=(1,2,7,-4) \oplus \mathfrak{u}=1,2,7,-4,5,10,3,12,16,6,18,8,13,20,15,9,17,11,}$ $21,14,19,22,23,24$

18. $\mathfrak{l}=\mathfrak{h} \oplus \pm(1) \oplus \mathfrak{u}=2,7,-4,5,10,3,12,16,6,18,8,13,20,15,9,17,11,21,14$, $19,22,23,24$

19. $\mathfrak{l}=\mathfrak{h} \oplus \pm(2) \oplus \mathfrak{u}=1,7,-4,5,10,3,12,16,6,18,8,13,20,15,9,17,11,21,14$, $19,22,23,24$

$20 . \mathfrak{l}=\mathfrak{h} \oplus \pm(7) \oplus \mathfrak{u}=1,2,-4,5,10,3,12,16,6,18,8,13,20,15,9,17,11,21,14$, $19,22,23,24$

21. $\mathfrak{l}=\mathfrak{h} \oplus \pm(1,2,5) \oplus \mathfrak{u}=7,-4,10,3,12,16,6,18,8,13,20,15,9,17,11,21,14$, $\frac{19,22,23,24}{22 . \quad \mathfrak{l}=\mathfrak{h} \oplus \pm(1,7) \oplus \mathfrak{u}=2,-4,5,10,3,12,16,6,18,8,13,20,15,9,17,11,21,14,}$ $19,22,23,24$

$23 . \mathfrak{l}=\mathfrak{h} \oplus \pm(2,7,10,16) \oplus \mathfrak{u}=1,-4,5,3,12,6,18,8,13,20,15,9,17,11,21,14$, $19,22,23,24$

24. $\mathfrak{l}=\mathfrak{h} \oplus \pm(1,2,7,5,10,12,16,18,20) \oplus \mathfrak{u}=-4,3,6,8,13,15,9,17,11,21,14$, $19,22,23,24$

$25 . \mathfrak{h}=(1,16,-7,3) \oplus \mathfrak{u}=1,16,-7,3,18,10,-4,12,2,13,5,15,6,20,8,9,21$, $11,17,22,19,14,23,24$

$26 . \quad \mathfrak{l}=\mathfrak{h} \oplus \pm(1) \oplus \mathfrak{u}=16,-7,3,18,10,-4,12,2,13,5,15,6,20,8,9,21,11,17$, $22,19,14,23,24$

$27 . \mathfrak{l}=\mathfrak{h} \oplus \pm(16) \oplus \mathfrak{u}=1,-7,3,18,10,-4,12,2,13,5,15,6,20,8,9,21,11,17$, $22,19,14,23,24$ 
$28 . \quad \mathfrak{l}=\mathfrak{h} \oplus \pm(3) \oplus \mathfrak{u}=1,16,-7,18,10,-4,12,2,13,5,15,6,20,8,9,21,11,17$ $22,19,14,23,24$

$29 . \quad \mathfrak{l}=\mathfrak{h} \oplus \pm(1,16,18) \oplus \mathfrak{u}=-7,3,10,-4,12,2,13,5,15,6,20,8,9,21,11,17$, $22,19,14,23,24$

$30 . \quad \mathfrak{l}=\mathfrak{h} \oplus \pm(1,3) \oplus \mathfrak{u}=16,-7,18,10,-4,12,2,13,5,15,6,20,8,9,21,11,17$, $22,19,14,23,24$

$31 . \quad \mathfrak{l}=\mathfrak{h} \oplus \pm(16,3) \oplus \mathfrak{u}=1,-7,18,10,-4,12,2,13,5,15,6,20,8,9,21,11,17$, $22,19,14,23,24$

$32 . \quad \mathfrak{l}=\mathfrak{h} \oplus \pm(1,16,18,3) \oplus \mathfrak{u}=-7,10,-4,12,2,13,5,15,6,20,8,9,21,11,17$, $22,19,14,23,24$

$E_{6}$.

Proposition 3.7. Let $\mathfrak{g}_{\mathbb{C}}=E_{6}$ and fix a special nilpotent orbit $\mathcal{O}$ of $G_{\mathbb{C}}$ on $\mathfrak{g}_{\mathbb{C}}$. Then there exists a real form $G$ such that some irreducible component of $\mathcal{O} \cap \mathfrak{p}_{\mathbb{C}}$ is a Richardson orbit of $K_{\mathbb{C}}$ on the nilpotent cone of $\mathfrak{p}_{\mathbb{C}}$.

Proof. We order the roots of $E_{6}$ as in the table below, and we use the Bourbaki system of simple roots $\Delta=\left\{\alpha_{1}, \alpha_{2}, \alpha_{3}, \alpha_{4}, \alpha_{5}, \alpha_{6}\right\}$. The Cartan involution $\theta$ with +1 -eigenspace $\mathfrak{k}$ and -1 -eigenspace $\mathfrak{p}$ depends on the real forms. If $\mathfrak{g}_{\mathbb{R}}=E I I$, then $\mathfrak{k}_{\mathbb{C}}=\mathfrak{s l}_{6}(\mathbb{C}) \oplus \mathfrak{s l}_{2}(\mathbb{C})$ and the vector space $\mathfrak{p}_{\mathbb{C}}$ is the complex span of nonzero root vectors $X_{\beta}$, where $\beta=c_{1} \alpha_{1}+c_{2} \alpha_{2}+c_{3} \alpha_{3}+c_{4} \alpha_{4}+c_{5} \alpha_{5}+c_{6} \alpha_{6}$ with $c_{2}= \pm 1$. If $\mathfrak{g}_{\mathbb{R}}=E I I I$, then $\mathfrak{k}_{\mathbb{C}}=\mathfrak{s o}_{10}(\mathbb{C}) \oplus \mathbb{C}$ and the vector space $\mathfrak{p}_{\mathbb{C}}$ is the complex span of nonzero root vectors $X_{\beta}$, where $\beta=c_{1} \alpha_{1}+c_{2} \alpha_{2}+c_{3} \alpha_{3}+c_{4} \alpha_{4}+c_{5} \alpha_{5}+c_{6} \alpha_{6}$ with $c_{6}= \pm 1$. If $\mathfrak{g}_{\mathbb{R}}=E I V$, then $\mathfrak{k}_{\mathbb{C}}=F_{4}$. In this case there are no noncompact imaginary roots. The compact imaginary roots are:

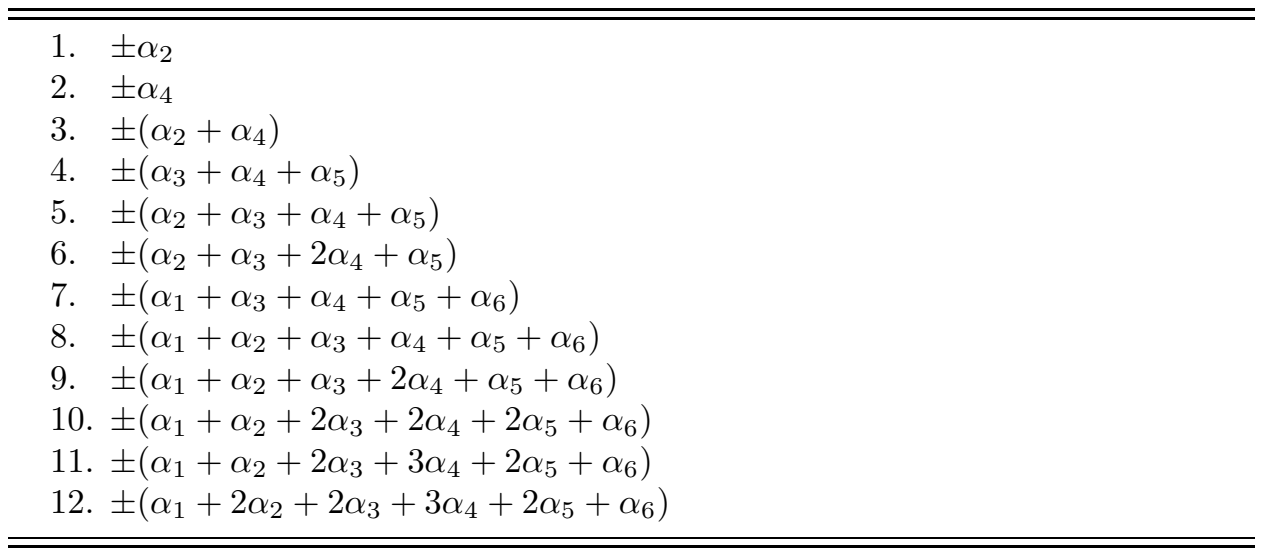

while the other roots are complex and $\mathfrak{g}_{\mathbb{C}}=\mathfrak{k}_{\mathbb{C}} \oplus \mathfrak{p}_{\mathbb{C}}$ with

$$
\begin{gathered}
\mathfrak{k}_{\mathbb{C}}=\mathfrak{t}_{\mathbb{C}} \oplus \sum_{\alpha \text { compact imaginary }} \mathbb{C} X_{\alpha} \bigoplus \sum_{(\alpha, \theta \alpha)} \sum_{\text {complex pairs }} \mathbb{C}\left(X_{\alpha}+\theta\left(X_{\alpha}\right)\right), \\
\mathfrak{p}_{\mathbb{C}}=\mathfrak{s}_{\mathbb{C}} \oplus \bigoplus_{(\alpha, \theta \alpha)} \sum_{\text {complex pairs }} \mathbb{C}\left(X_{\alpha}-\theta\left(X_{\alpha}\right)\right) .
\end{gathered}
$$

Here $X_{\alpha}$ is a nonzero vector of the root space $\mathfrak{g}_{\mathrm{C}}^{\alpha}$. An imaginary root $\alpha$ is compact (noncompact) if its root space $\mathfrak{g}_{\mathbb{C}}^{\alpha}$ lies in $\mathfrak{k}_{\mathbb{C}}\left(\mathfrak{p}_{\mathbb{C}}\right)$. The fundamental Cartan subalgebra is $\mathfrak{h}_{\mathbb{C}}=\mathfrak{t}_{\mathbb{C}} \oplus \mathfrak{s}_{\mathbb{C}}$. See [2] for more details. 


\begin{tabular}{lll}
\hline & \multicolumn{2}{c}{ Positive roots of $E_{6}$} \\
\hline 1. $[1,0,0,0,0,0]$ & $13 .[0,1,1,1,0,0]$ & $25 .[0,1,1,1,1,1]$ \\
2. $[0,1,0,0,0,0]$ & $14 .[0,1,0,1,1,0]$ & $26 .[1,1,1,2,1,0]$ \\
3. $[0,0,1,0,0,0]$ & $15 .[0,0,1,1,1,0]$ & $27 .[1,1,1,1,1,1]$ \\
4. $[0,0,0,1,0,0]$ & $16 .[0,0,0,1,1,1]$ & $28 .[0,1,1,2,1,1]$ \\
5. $[0,0,0,0,1,0]$ & 17. $[1,1,1,1,0,0]$ & $29 .[1,1,2,2,1,0]$ \\
6. $[0,0,0,0,0,1]$ & 18. $[1,0,1,1,1,0]$ & $30 .[1,1,1,2,1,1]$ \\
7. $[1,0,1,0,0,0]$ & $19 .[0,1,1,1,1,0]$ & $31 .[0,1,1,2,2,1]$ \\
8. $[0,1,0,1,0,0]$ & $20 .[0,1,0,1,1,1]$ & $32 .[1,1,2,2,1,1]$ \\
9. $[0,0,1,1,0,0]$ & $21 .[0,0,1,1,1,1]$ & $33 .[1,1,1,2,2,1]$ \\
10. $[0,0,0,1,1,0]$ & $22 .[1,1,1,1,1,0]$ & $34 .[1,1,2,2,2,1]$ \\
$11 .[0,0,0,0,1,1]$ & $23 .[1,0,1,1,1,1]$ & $35 .[1,1,2,3,2,1]$ \\
12. $[1,0,1,1,0,0]$ & $24 .[0,1,1,2,1,0]$ & $36 .[1,2,2,3,2,1]$ \\
\hline \hline
\end{tabular}

As before it is enough to consider noneven special orbits in $E_{6}$. The next table shows which of them are polarizable and therefore, by Tauvel's lemma, satisfy the proposition.

\section{Polarizable orbits of $E_{6}$}

Bala-Carter Label: $A_{2} \oplus 2 A_{1}$

Real form: EII

Root vectors for $e: 27,-2,31,29$

Parabolic: Levi-Type: $A_{4}+A_{1}$

Cartan subalgebra: $1,4,13,5,-14,20$

Roots vectors for Levi: $\pm(1,13,5,4,17,19,10,22,24,26) \pm(20)$

Roots vectors for nilradical: $-14,-8,6,-2,3,11,7,9,16,25,12,27,15,28,18$, $30,31,29,33,21,36,23,32,34,35$

Bala-Carter Label: $A_{3}$

Special

Real form: EII

Root vectors for $e:-2,24,27$

Parabolic: Levi-Type: $A_{4}$

$\operatorname{dim} \mathfrak{g}_{\mathbb{C}}^{e}=26$

Cartan subalgebra: $1,-2,3,8,5,6$

Roots vectors for Levi: $\pm(1,3,8,5,7,13,14,17,19,22)$

Roots vectors for nilradical: $-2,6,4,11,9,10,20,12,15,16,25,18,27,24,21$,

$26,23,28,29,30,31,32,33,34,36,35$

Bala-Carter Label: $A_{4} \oplus A_{1}$

Special

Real form: EII

Root vectors for $e: 27,28,29,-17,-19$

Parabolic: Levi-Type: $A_{2} \oplus A_{1} \oplus A_{1}$

Special

$\operatorname{dim} \mathfrak{g}_{\mathbb{C}}^{e}=28$

dimension $=53$

Cartan subalgebra: $-17,24,22,-19,3,20$

Roots vectors for Levi: $\pm(3,20,25), \pm(24), \pm(22)$

Roots vectors for nilradical: $-17,-19,5,4,1,-14,-13,26,9,7,6,10,-8,29$,

$28,27,15,11,12,36,-2,31,30,18,16,32,33,21,34,23,35$ 
Bala-Carter Label: $D_{5}\left(a_{1}\right)$

Real form: EII

Root vectors for $e$ : $-13,28,22,27,-14,-20$

$\operatorname{dim} \mathfrak{g}_{\mathrm{C}}^{e}=14$

Parabolic: Levi-Type: $A_{2} \oplus A_{1}$

Cartan subalgebra: $17,4,-13,19,6,-20$

Roots vectors for Levi: $\pm(19,4,24), \pm(17)$

Roots vectors for nilradical: $-13,6,-20,1,5,25,-14,22,10,28,11,3,26,27$,

$16,9,-8,30,7,31,-2,36,12,15,33,29,21,18,32,23,34,35$

We shall now deal with the remaining orbits of interest. They are labeled as follows: $A_{1}, 2 A_{1}, A_{2}+A_{1}$. In each case we will exhibit a real form and a theta stable parabolic subalgebra $\mathfrak{q}=\mathfrak{l} \oplus \mathfrak{u}$ such that the given orbit intersect $\mathfrak{u} \cap \mathfrak{p}_{\mathbb{C}}$ in an open dense set.

In the case of $A_{1}$ we see below that $\mathfrak{u} \cap \mathfrak{p}_{\mathbb{C}}=\mathbb{C}\left(X_{32}-X_{33}\right)$. Hence the $K_{\mathbb{C}}$-orbit of $\left(X_{32}-X_{33}\right)$ is the $K_{\mathbb{C}}$-saturation of $\mathfrak{u} \cap \mathfrak{p}_{\mathbb{C}}$.

Bala-Carter Label: $A_{1}$

Special

Real form: EIV

Root vectors for $e$ : 32,33

Parabolic: Levi-Type: $D_{5}$

Cartan subalgebra: $1,2,3,4,5,6$

Roots vectors for Levi: $\pm(6,5,4,2,3,11,10,8,9,16,14,15,13,20$,

$21,19,25,24,28,31,1,7,12,17,18,22,26,29)$

Roots vectors for nilradical: $23,27,30,32,33,34,35,36$

In the case of the orbit labeled $2 A_{1}$ we use the real form $E I I$ and a theta-stable parabolic subalgebra $\mathfrak{q}=\mathfrak{l} \oplus \mathfrak{u}$ such that the Cartan product of all roots in $\mathfrak{u} \cap \mathfrak{p}_{\mathbb{C}}$ is nonnegative an there are no instances of three orthogonal roots.

Bala-Carter Label: $2 A_{1}$

Real form: EII

Root vectors for $e: 20,32$

Parabolic: Levi-Type: $D_{5}$

Cartan subalgebra: $1,2,3,4,5,6$

Roots vectors for Levi: $\pm(1,3,4,2,5,7,9,8,10,12,13,15,14,17,18,19$, $22,24,26,29)$

Roots vectors for nilradical: $6,11,16,20,21,23,25,27,28$, $30,31,32,33,34,35,36$ 
In the case of the orbit labeled $A_{2}+A_{1}$ we use the real form EIII and a thetastable parabolic subalgebra $\mathfrak{q}=\mathfrak{l} \oplus \mathfrak{u}$ such that $\mathfrak{u} \cap \mathfrak{p}_{\mathbb{C}}$ contains no representatives of the orbits labeled $2 A_{2}$ or $A_{3}$. But we can find a representative of $A_{2}+A_{1}$ in $\mathfrak{u} \cap \mathfrak{p}_{\mathbb{C}}$. This is the largest orbit intersecting $\mathfrak{u} \cap \mathfrak{p}_{\mathbb{C}}$.

Bala-Carter Label: $A_{2} \oplus A_{1}$

Special

Real form: EIII

Root vectors for $e$ : 23, $-6,36$

Parabolic: Levi-Type: $D_{4}$

Cartan subalgebra: $23,3,2,-25,28,5$

Roots vectors for Levi: $\pm(3,-25,28,2,-20,4,-21,9,-16,8,13,-11)$

Roots vectors for nilradical: $23,5,27,31,1,10,7,30,15,14,32,33,19,12,34$,

$-6,17,18,24,22,35,36,26,29$

This concludes the proof.

If $G_{\mathrm{C}}$ is of type $E_{7}$ or $E_{8}$, then the minimal orbit is not polarizable. This fact can be verified by showing that the dimension of each theta parabolic subalgebra will not satisfy the above definition. Moreover, all theta-stable parabolic subalgebras relative to each real form contain a nilpotent $A_{1}+A_{1}$. Hence no theta-stable parabolic subalgebra $\mathfrak{q}=\mathfrak{l} \oplus \mathfrak{u}$ will satisfy the analogue of Trapa's theorem. Again this fact can be checked easily using the algorithm given in [4].

$E_{7}$.

Proposition 3.8. Let $\mathfrak{g}_{\mathbb{C}}=E_{7}$ and fix a nonminimal special nilpotent orbit $\mathcal{O}$ of $G_{\mathbb{C}}$ on $\mathfrak{g}_{\mathbb{C}}$. Then there exists a real form $G$ such that some irreducible component of $\mathcal{O} \cap \mathfrak{p}_{\mathbb{C}}$ is a Richardson orbit of $K_{\mathbb{C}}$ on the nilpotent cone of $\mathfrak{p}_{\mathbb{C}}$.

Proof. We order the roots of $E_{7}$ as in the table below, and we use the Bourbaki system of simple roots

$$
\Delta=\left\{\alpha_{1}, \alpha_{2}, \alpha_{3}, \alpha_{4}, \alpha_{5}, \alpha_{6}, \alpha_{7}\right\} .
$$

The Cartan involution $\theta$ with +1 -eigenspace $\mathfrak{k}$ and -1 -eigenspace $\mathfrak{p}$ depends on the real forms. If $\mathfrak{g}_{\mathbb{R}}=E V$, then $\mathfrak{k}_{\mathbb{C}}=\mathfrak{s l}_{8}(\mathbb{C})$ and the vector space $\mathfrak{p}_{\mathbb{C}}$ is the complex span of nonzero root vectors $X_{\beta}$, where

$$
\beta=c_{1} \alpha_{1}+c_{2} \alpha_{2}+c_{3} \alpha_{3}+c_{4} \alpha_{4}+c_{5} \alpha_{5}+c_{6} \alpha_{6}+c_{7} \alpha_{7}
$$

with $c_{2}= \pm 1$. If $\mathfrak{g}_{\mathbb{R}}=E V I$, then $\mathfrak{k}_{\mathbb{C}}=\mathfrak{s o}_{12}(\mathbb{C}) \oplus \mathfrak{s l}_{2}(\mathbb{C})$, and the vector space $\mathfrak{p}_{\mathbb{C}}$ is the complex span of nonzero root vectors $X_{\beta}$, where

$$
\beta=c_{1} \alpha_{1}+c_{2} \alpha_{2}+c_{3} \alpha_{3}+c_{4} \alpha_{4}+c_{5} \alpha_{5}+c_{6} \alpha_{6}+c_{7} \alpha_{7}
$$

with $c_{1}= \pm 1$. If $\mathfrak{g}_{\mathbb{R}}=E V I I$, then $\mathfrak{k}_{\mathbb{C}}=\mathfrak{e}_{6}(\mathbb{C}) \oplus \mathbb{C}$ and the vector space $\mathfrak{p}_{\mathbb{C}}$ is the complex span of nonzero root vectors $X_{\beta}$, where

$$
\beta=c_{1} \alpha_{1}+c_{2} \alpha_{2}+c_{3} \alpha_{3}+c_{4} \alpha_{4}+c_{5} \alpha_{5}+c_{6} \alpha_{6}+c_{7} \alpha_{7}
$$

with $c_{7}= \pm 1$. 


\begin{tabular}{|c|c|c|}
\hline \multicolumn{3}{|c|}{ Positive roots of $E_{7}$} \\
\hline 1. $[1,0,0,0,0,0,0]$ & 22. $[0,1,1,1,1,0,0]$ & 43. $[1,1,1,2,2,1,0]$ \\
\hline 2. $[0,1,0,0,0,0,0]$ & 23. $[0,1,0,1,1,1,0]$ & 44. $[1,1,1,2,1,1,1]$ \\
\hline 3. $[0,0,1,0,0,0,0]$ & 24. $[0,0,1,1,1,1,0]$ & 45. $[0,1,1,2,2,1,1]$ \\
\hline 4. $[0,0,0,1,0,0,0]$ & 25. $[0,0,0,1,1,1,1]$ & 46. $[1,1,2,2,2,1,0]$ \\
\hline 5. $[0,0,0,0,1,0,0]$ & 26. $[1,1,1,1,1,0,0]$ & 47. $[1,1,2,2,1,1,1]$ \\
\hline 6. $[0,0,0,0,0,1,0]$ & 27. $[1,0,1,1,1,1,0]$ & 48. $[1,1,1,2,2,1,1]$ \\
\hline 7. $[0,0,0,0,0,0,1]$ & 28. $[0,1,1,2,1,0,0]$ & 49. $[0,1,1,2,2,2,1]$ \\
\hline 8. $[1,0,1,0,0,0,0]$ & 29. $[0,1,1,1,1,1,0]$ & 50. $[1,1,2,3,2,1,0]$ \\
\hline 9. $[0,1,0,1,0,0,0]$ & 30. $[0,1,0,1,1,1,1]$ & 51. $[1,1,2,2,2,1,1]$ \\
\hline 10. $[0,0,1,1,0,0,0]$ & 31. $[0,0,1,1,1,1,1]$ & 52. $[1,1,1,2,2,2,1]$ \\
\hline 11. $[0,0,0,1,1,0,0]$ & 32. $[1,1,1,2,1,0,0]$ & 53. $[1,2,2,3,2,1,0]$ \\
\hline 12. $[0,0,0,0,1,1,0]$ & 33. $[1,1,1,1,1,1,0]$ & 54. $[1,1,2,3,2,1,1]$ \\
\hline 13. $[0,0,0,0,0,1,1]$ & 34. $[1,0,1,1,1,1,1]$ & 55. $[1,1,2,2,2,2,1]$ \\
\hline 14. $[1,0,1,1,0,0,0]$ & 35. $[0,1,1,2,1,1,0]$ & 56. $[1,2,2,3,2,1,1]$ \\
\hline 15. $[0,1,1,1,0,0,0]$ & 36. $[0,1,1,1,1,1,1]$ & 57. $[1,1,2,3,2,2,1]$ \\
\hline 16. $[0,1,0,1,1,0,0]$ & 37. $[1,1,2,2,1,0,0]$ & 58. $[1,2,2,3,2,2,1]$ \\
\hline 17. $[0,0,1,1,1,0,0]$ & 38. $[1,1,1,2,1,1,0]$ & 59. $[1,1,2,3,3,2,1]$ \\
\hline 18. $[0,0,0,1,1,1,0]$ & 39. $[1,1,1,1,1,1,1]$ & 60. $[1,2,2,3,3,2,1]$ \\
\hline 19. $[0,0,0,0,1,1,1]$ & 40. $[0,1,1,2,2,1,0]$ & 61. $[1,2,2,4,3,2,1]$ \\
\hline 20. $[1,1,1,1,0,0,0]$ & 41. $[0,1,1,2,1,1,1]$ & 62. $[1,2,3,4,3,2,1]$ \\
\hline 21. $[1,0,1,1,1,0,0]$ & 42. $[1,1,2,2,1,1,0]$ & 63. $[2,2,3,4,3,2,1]$ \\
\hline
\end{tabular}

As before it is enough to consider noneven special orbits in $E_{7}$. The next table shows which of them are polarizable and therefore by Tauvel's lemma satisfy the proposition.

\section{Polarizable orbits of $E_{7}$}

Bala-Carter Label: $D_{4}\left(a_{1}\right) \oplus A_{1}$

Real form: $\mathrm{EV}$

Root vectors for $e: 37,-16,43,38,45,41 \quad \operatorname{dim} \mathfrak{g}_{\mathrm{C}}^{e}=37$

Parabolic: Levi-Type: $A_{5}$

dimension $=85$

Cartan subalgebra: $1,4,15,5,6,7,-30$

Roots vectors for Levi: $\pm(1,15,5,6,7,20,22,12,13,26,29,19,33,36,39)$

Roots vectors for nilradical: $4,-30,11,-23,28,18,-16,32,35,25,-9,38,40,41$, $-2,3,43,44,8,45,10,53,48,14,49,17,56,52,21,24,58,37,27,31,60,42,34,61$, $46,47,50,51,54,55,57,59,62,63$

Bala-Carter Label: $A_{4} \oplus A_{1}$

Real form: $\mathrm{EV}$

Root vectors for $e: 26,38,45,-15,-16$

Parabolic: Levi-Type: $A_{4} \oplus A_{1}$ Special

Cartan subalgebra: $20,-28,5,4,3,23,7$

Roots vectors for Levi: $\pm(7,23,3,4,30,29,10,36,35,41) \pm(20)$

Roots vectors for nilradical: $-28,5,26,-22,11,32,-15,-16,17,1,37,-9,6,40$, $8,53,-2,12,13,45,14,33,56,18,19,21,38,39,24,25,43,42,44,31,46,48$, $47,49,50,51,58,27,54,60,34,61,52,62,55,57,59,63$ 


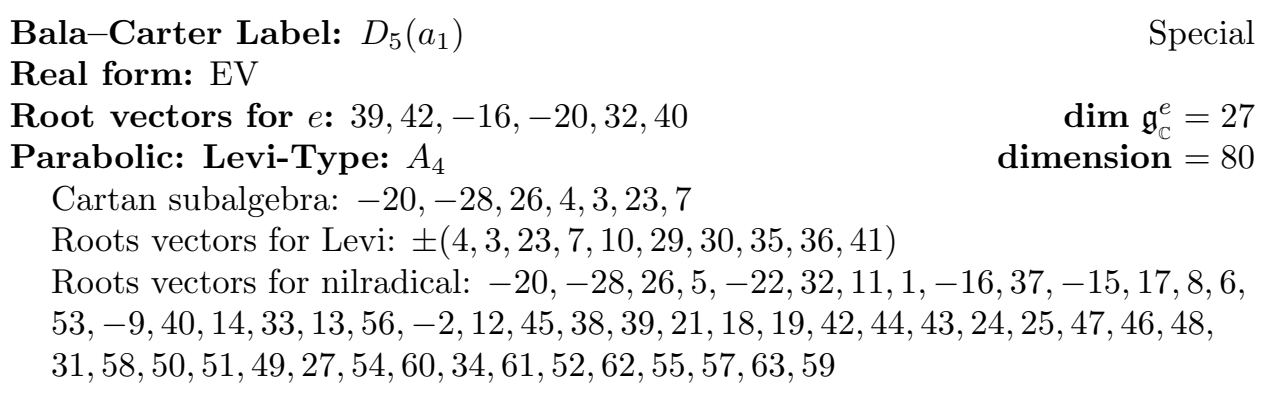

Bala-Carter Label: $D_{5} \oplus A_{1}$

Special

Real form: EV

Root vectors for $e$ : $32,33,41,-20,-22,-23$

Parabolic: Levi-Type: $A_{2} \oplus A_{2}$

Cartan subalgebra: $-20,28,26,6,-29,3,30$

Roots vectors for Levi: $\pm(28,6,35) \pm(3,30,36)$

Roots vectors for nilradical: $-20,26,-29,5,33,-22,-23,12,53,4,1,-16,7,40$, $-15,32,10,8,13,11,-9,38,37,41,39,18,17,19,42,56,43,24,45,14,58,46,-2$, $49,44,21,60,25,47,27,48,31,50,52,51,61,55,62,34,54,57,63,59$

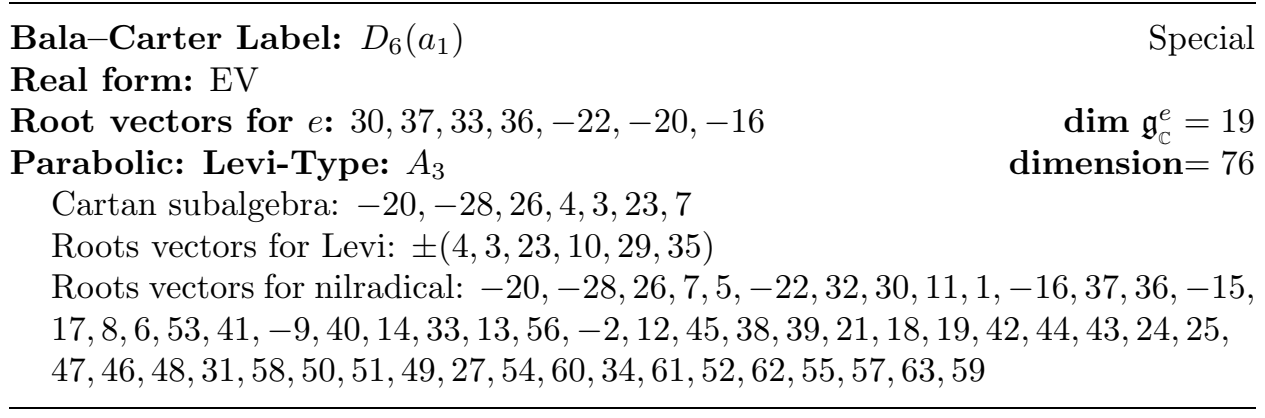

We shall now deal with the remaining orbits of interest. In each case we will exhibit a real form and a theta stable parabolic subalgebra $\mathfrak{q}=\mathfrak{l} \oplus \mathfrak{u}$, such that the given orbit intersect $\mathfrak{u} \cap \mathfrak{p}_{\mathbb{C}}$ in an open dense set.

In the case of the orbit labeled $2 A_{1}$, we use the real form EVII and a thetastable parabolic subalgebra $\mathfrak{q}=\mathfrak{l} \oplus \mathfrak{u}$ such that the Cartan product of all roots in $\mathfrak{u} \cap \mathfrak{p}_{\mathbb{C}}$ is nonnegative, and there are no instances of three orthogonal roots.

\begin{tabular}{l}
\hline \hline Bala-Carter Label: $2 A_{1}$ \\
Real form: EVII \\
Root vectors for $e: 34,56$ \\
Parabolic: Levi-Type: $D_{6}$ \\
$\quad$ Cartan subalgebra: $1,2,3,4,5,6,7$ \\
$\quad$ Roots vectors for Levi: $\pm(76,5,4,2,3,13,12,11,9,10,19,18,16,17$, \\
$\quad 15,25,23,24,22,30,31,29,28,36,35,41,40,45,49)$ \\
$\quad$ Roots vectors for nilradical: $18,14,20,21,26,27,32,33,34,37,38,39,42$, \\
$\quad 43,44,46,47,48,50,51,52,53,54,55,56,57,58,59,60,61,62,63$ \\
\hline
\end{tabular}


In the case of the orbit labeled $A_{2}+A_{1}$, we use the real form $E V I I$ and a thetastable parabolic subalgebra $\mathfrak{q}=\mathfrak{l} \oplus \mathfrak{u}$ such that $\mathfrak{u} \cap \mathfrak{p}_{\mathbb{C}}$ contains no representatives of the orbits labeled $2 A_{2}$ or $A_{3}$. But we can find a representative of $A_{2}+A_{1}$ in $\mathfrak{u} \cap \mathfrak{p}_{\mathbb{C}}$. This is the largest orbit intersecting $\mathfrak{u} \cap \mathfrak{p}_{\mathbb{C}}$.

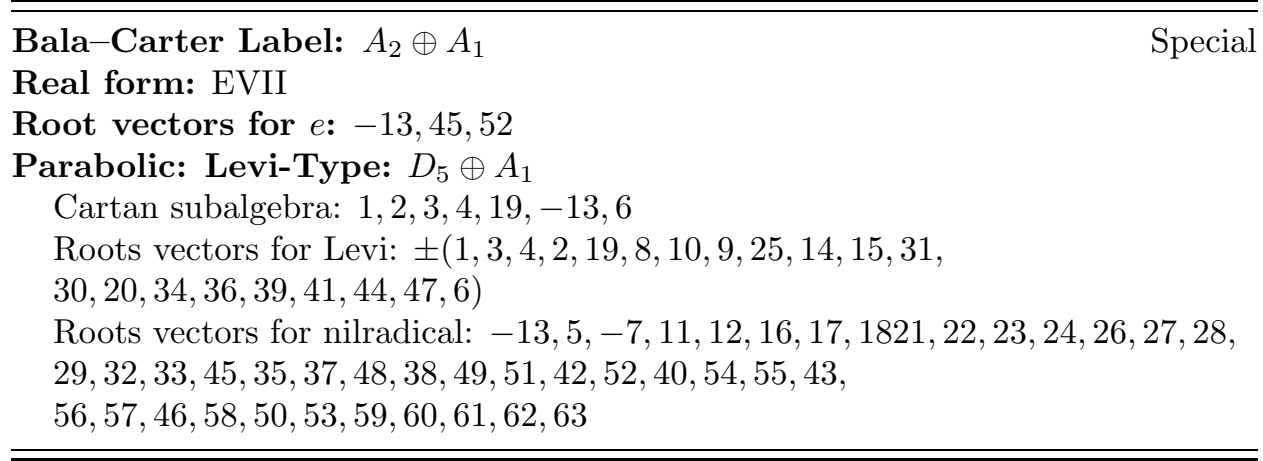

In the case of the orbit labeled $A_{2}+2 A_{1}$, we use the real form $E V I$ and a thetastable parabolic subalgebra $\mathfrak{q}=\mathfrak{l} \oplus \mathfrak{u}$ such that $\mathfrak{u} \cap \mathfrak{p}_{\mathbb{C}}$ contains no representatives of the orbits labeled $2 A_{2}$ or $A_{3}$. But we can find a representative of $A_{2}+2 A_{1}$ in $\mathfrak{u} \cap \mathfrak{p}_{\mathbb{C}}$. This is the largest orbit intersecting $\mathfrak{u} \cap \mathfrak{p}_{\mathbb{C}}$.

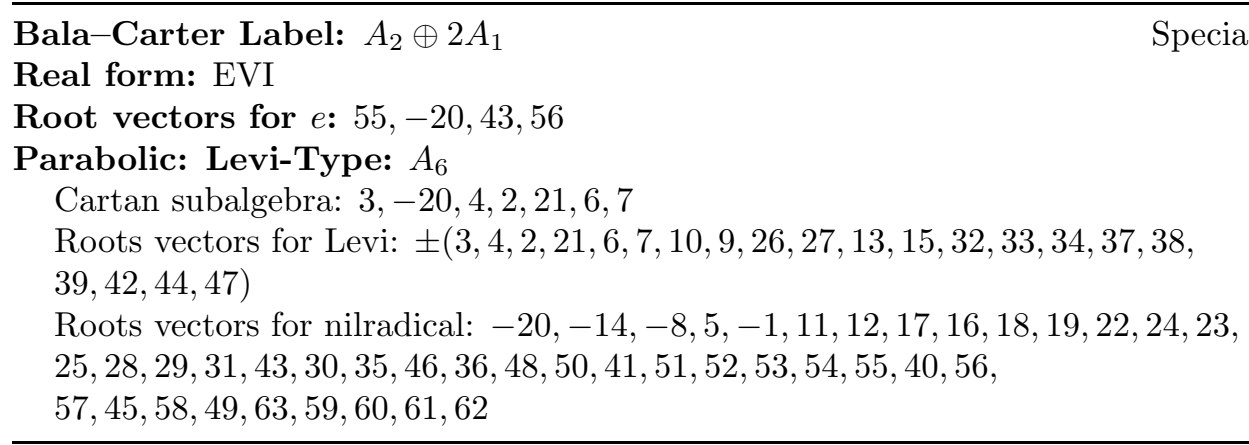

In the case orbit labeled $A_{3}$ we use the real form EVII and a theta-stable parabolic subalgebra $\mathfrak{q}=\mathfrak{l} \oplus \mathfrak{u}$ such that $\mathfrak{u} \cap \mathfrak{p}_{\mathbb{C}}$ contains no representatives of the orbits labeled $2 A_{2}$ or $A_{3}+A_{1}$ or $A_{4}$. But we can find a representative of $A_{3}$ in $\mathfrak{u} \cap \mathfrak{p}_{\mathbb{C}}$. This is the largest orbit intersecting $\mathfrak{u} \cap \mathfrak{p}_{\mathbb{C}}$.

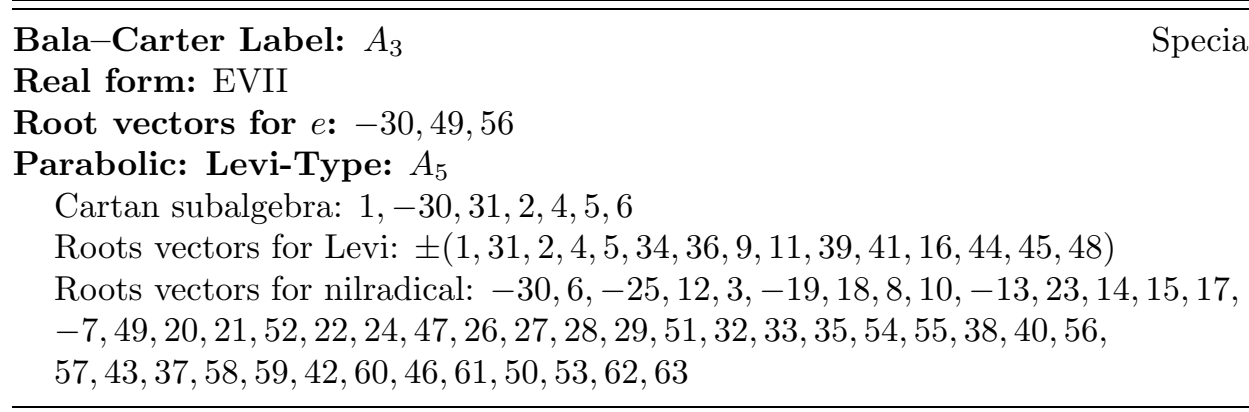


Finally for the orbit labeled $A_{3}+A_{2}$, we use the real form $E V I$ and a thetastable parabolic subalgebra $\mathfrak{q}=\mathfrak{l} \oplus \mathfrak{u}$ such that $\mathfrak{u} \cap \mathfrak{p}_{\mathbb{C}}$ contains no representatives of the orbits labeled $2 A_{3}$ or $A_{4}$ or any higher-dimensional orbits. But we can find a representative of $A_{3}+A_{2}$ in $\mathfrak{u} \cap \mathfrak{p}_{\mathbb{C}}$. This is the largest orbit intersecting $\mathfrak{u} \cap \mathfrak{p}_{\mathbb{C}}$.

Bala-Carter Label: $A_{3} \oplus 2 A_{2}$

Real form: EVI

Root vectors for $e: 50,55,-33,-21,48$

Parabolic: Levi-Type: $A_{4} \oplus A_{2}$

Cartan subalgebra: $3,5,32,6,-33,2,34$

Roots vectors for Levi: $\pm(3,32,6,5,37,38,12,42,43,46,2,34,39)$

Roots vectors for nilradical: $-33,-26,-27,-20,4,-21,7,10,11,-14,9,13,17$, $15,18,16,19,44,24,22,47,23,48,50,29,51,-8,52,53,-1,55,25,28$,

$63,31,30,35,54,36,40,57,56,59,58,60,41,45,49,61,62$

This concludes the proof.

$E_{8}$.

All theta-stable parabolic subalgebras relative to the two real forms of $E_{8}$ contains a representative of the nilpotent orbit labeled $3 A_{1}$. Hence $2 A_{1}$ cannot be the $K_{\mathbb{C}}$-saturation or any $\mathfrak{q}$. However the next proposition shows that most special orbits of $E_{8}$ are Richardson.

Proposition 3.9. Let $\mathfrak{g}_{\mathbb{C}}=E_{8}$ and fix a special nilpotent orbit $\mathcal{O}$ of $G_{\mathbb{C}}$ on $\mathfrak{g}_{\mathbb{C}}$ such that $\mathcal{O}$ is either even or is one of the orbits given below. Then there exists a real form $G$ such that some irreducible component of $\mathcal{O} \cap \mathfrak{p}_{\mathbb{C}}$ is a Richardson orbit of $K_{\mathbb{C}}$ on the nilpotent cone of $\mathfrak{p}_{\mathbb{C}}$.

Proof. We order the roots of $E_{8}$ as in the table below, and we use the Bourbaki system of simple roots

$$
\Delta=\left\{\alpha_{1}, \alpha_{2}, \alpha_{3}, \alpha_{4}, \alpha_{5}, \alpha_{6}, \alpha_{7}, \alpha_{8}\right\}
$$

The Cartan involution $\theta$ with +1 -eigenspace $\mathfrak{k}$ and -1 -eigenspace $\mathfrak{p}$ depends on the real forms. If $\mathfrak{g}_{\mathbb{R}}=E V I I I$, then $\mathfrak{k}_{\mathbb{C}}=\mathfrak{s o}_{16}(\mathbb{C})$ and the vector space $\mathfrak{p}_{\mathbb{C}}$ is the complex span of nonzero root vectors $X_{\beta}$, where

$$
\beta=c_{1} \alpha_{1}+c_{2} \alpha_{2}+c_{3} \alpha_{3}+c_{4} \alpha_{4}+c_{5} \alpha_{5}+c_{6} \alpha_{6}+c_{7} \alpha_{7}+c_{8} \alpha_{8}
$$

with $c_{1}= \pm 1$. 


\begin{tabular}{|c|c|c|}
\hline \multicolumn{3}{|c|}{ Positive roots of $E_{8}$} \\
\hline 1. $[1,0,0,0,0,0,0,0]$ & 41. $[0,1,1,1,1,1,1,0]$ & 81. $[1,2,2,3,2,1,1,1]$ \\
\hline 2. $[0,1,0,0,0,0,0,0]$ & 42. $[0,1,0,1,1,1,1,1]$ & 82. $[1,1,2,3,3,2,1,0]$ \\
\hline 3. $[0,0,1,0,0,0,0,0]$ & 43. $[0,0,1,1,1,1,1,1]$ & 83. $[1,1,2,3,2,2,1,1]$ \\
\hline 4. $[0,0,0,1,0,0,0,0]$ & 44. $[1,1,2,2,1,0,0,0]$ & 84. $[1,1,2,2,2,2,2,1]$ \\
\hline 5. $[0,0,0,0,1,0,0,0]$ & 45. $[1,1,1,2,1,1,0,0]$ & 85. $[1,2,2,3,3,2,1,0]$ \\
\hline 6. $[0,0,0,0,0,1,0,0]$ & 46. $[1,1,1,1,1,1,1,0]$ & 86. $[1,2,2,3,2,2,1,1]$ \\
\hline 7. $[0,0,0,0,0,0,1,0]$ & 47. $[1,0,1,1,1,1,1,1]$ & 87. $[1,1,2,3,3,2,1,1]$ \\
\hline 8. $[0,0,0,0,0,0,0,1]$ & 48. $[0,1,1,2,2,1,0,0]$ & 88. $[1,1,2,3,2,2,2,1]$ \\
\hline 9. $[1,0,1,0,0,0,0,0]$ & 49. $[0,1,1,2,1,1,1,0]$ & 89. $[1,2,2,4,3,2,1,0]$ \\
\hline 10. $[0,1,0,1,0,0,0,0]$ & 50. $[0,1,1,1,1,1,1,1]$ & 90. $[1,2,2,3,3,2,1,1]$ \\
\hline 11. $[0,0,1,1,0,0,0,0]$ & 51. $[1,1,2,2,1,1,0,0]$ & 91. $[1,2,2,3,2,2,2,1]$ \\
\hline 12. $[0,0,0,1,1,0,0,0]$ & 52. $[1,1,1,2,2,1,0,0]$ & 92. $[1,1,2,3,3,2,2,1]$ \\
\hline 13. $[0,0,0,0,1,1,0,0]$ & 53. $[1,1,1,2,1,1,1,0]$ & 93. $[1,2,3,4,3,2,1,0]$ \\
\hline 14. $[0,0,0,0,0,1,1,0]$ & 54. $[1,1,1,1,1,1,1,1]$ & 94. $[1,2,2,4,3,2,1,1]$ \\
\hline 15. $[0,0,0,0,0,0,1,1]$ & 55. $[0,1,1,2,2,1,1,0]$ & 95. $[1,2,2,3,3,2,2,1]$ \\
\hline 16. $[1,0,1,1,0,0,0,0]$ & 56. $[0,1,1,2,1,1,1,1]$ & 96. $[1,1,2,3,3,3,2,1]$ \\
\hline 17. $[0,1,1,1,0,0,0,0]$ & 57. $[1,1,2,2,2,1,0,0]$ & 97. $[2,2,3,4,3,2,1,0]$ \\
\hline 18. $[0,1,0,1,1,0,0,0]$ & 58. $[1,1,2,2,1,1,1,0]$ & 98. $[1,2,3,4,3,2,1,1]$ \\
\hline 19. $[0,0,1,1,1,0,0,0]$ & 59. $[1,1,1,2,2,1,1,0]$ & 99. $[1,2,2,4,3,2,2,1]$ \\
\hline 20. $[0,0,0,1,1,1,0,0]$ & 60. $[1,1,1,2,1,1,1,1]$ & 100. $[1,2,2,3,3,3,2,1]$ \\
\hline 21. $[0,0,0,0,1,1,1,0]$ & 61. $[0,1,1,2,2,2,1,0]$ & 101. $[2,2,3,4,3,2,1,1]$ \\
\hline $22 .[0,0,0,0,0,1,1,1]$ & 62. $[0,1,1,2,2,1,1,1]$ & 102. $[1,2,3,4,3,2,2,1]$ \\
\hline 23. $[1,1,1,1,0,0,0,0]$ & 63. $[1,1,2,3,2,1,0,0]$ & 103. $[1,2,2,4,3,3,2,1]$ \\
\hline 24. $[1,0,1,1,1,0,0,0]$ & 64. $[1,1,2,2,2,1,1,0]$ & 104. $[2,2,3,4,3,2,2,1]$ \\
\hline $25 .[0,1,1,1,1,0,0,0]$ & 65. $[1,1,2,2,1,1,1,1]$ & 105. $[1,2,3,4,3,3,2,1]$ \\
\hline $26 .[0,1,0,1,1,1,0,0]$ & 66. $[1,1,1,2,2,2,1,0]$ & 106. $[1,2,2,4,4,3,2,1]$ \\
\hline 27. $[0,0,1,1,1,1,0,0]$ & 67. $[1,1,1,2,2,1,1,1]$ & 107. $[2,2,3,4,3,3,2,1]$ \\
\hline 28. $[0,0,0,1,1,1,1,0]$ & 68. $[0,1,1,2,2,2,1,1]$ & 108. $[1,2,3,4,4,3,2,1]$ \\
\hline $29 .[0,0,0,0,1,1,1,1]$ & 69. $[1,2,2,3,2,1,0,0]$ & 109. $[2,2,3,4,4,3,2,1]$ \\
\hline $30 .[1,1,1,1,1,0,0,0]$ & 70. $[1,1,2,3,2,1,1,0]$ & 110. $[1,2,3,5,4,3,2,1]$ \\
\hline 31. $[1,0,1,1,1,1,0,0]$ & 71. $[1,1,2,2,2,2,1,0]$ & 111. $[2,2,3,5,4,3,2,1]$ \\
\hline 32. $[0,1,1,2,1,0,0,0]$ & 72. $[1,1,2,2,2,1,1,1]$ & 112. $[1,3,3,5,4,3,2,1]$ \\
\hline 33. $[0,1,1,1,1,1,0,0]$ & 73. $[1,1,1,2,2,2,1,1]$ & 113. $[2,3,3,5,4,3,2,1]$ \\
\hline 34. $[0,1,0,1,1,1,1,0]$ & 74. $[0,1,1,2,2,2,2,1]$ & 114. $[2,2,4,5,4,3,2,1]$ \\
\hline $35 .[0,0,1,1,1,1,1,0]$ & 75. $[1,2,2,3,2,1,1,0]$ & $115 .[2,3,4,5,4,3,2,1]$ \\
\hline 36. $[0,0,0,1,1,1,1,1]$ & 76. $[1,1,2,3,2,2,1,0]$ & 116. $[2,3,4,6,4,3,2,1]$ \\
\hline 37. $[1,1,1,2,1,0,0,0]$ & 77. $[1,1,2,3,2,1,1,1]$ & 117. $[2,3,4,6,5,3,2,1]$ \\
\hline 38. $[1,1,1,1,1,1,0,0]$ & 78. $[1,1,2,2,2,2,1,1]$ & 118. $[2,3,4,6,5,4,2,1]$ \\
\hline 39. $[1,0,1,1,1,1,1,0]$ & 79. $[1,1,1,2,2,2,2,1]$ & 119. $[2,3,4,6,5,4,3,1]$ \\
\hline 40. $[0,1,1,2,1,1,0,0]$ & 80. $[1,2,2,3,2,2,1,0]$ & 120. $[2,3,4,6,5,4,3,2]$ \\
\hline
\end{tabular}


As before it is enough to consider noneven special orbits in $E_{8}$. The next table shows which of them are polarizable and therefore by Tauvel's lemma satisfy the proposition.

\section{Polarizable orbits of $E_{8}$}

Bala-Carter Label: $A_{4} \oplus A_{2} \oplus A_{1}$

Real form: EVIII

Root vectors for $e$ : $63,71,72,73,75,-16,-30$

Parabolic: Levi-Type: $A_{6} \oplus A_{1}$

Cartan subalgebra: $44,5,-37,4,2,31,7,8$

Roots vectors for Levi: $\pm(8,7,31,2,4,5,15,39,38,10,12,47,46,45,18,54,53$, $52,60,59,67), \pm(44)$

Roots vectors for nilradical: $-37,3,-30,11,-23,-24,19,17,-16,6$, $25,51,-9,13,14,32,57,58,20,21,22,-1,63,64,65,26,28,29,27,69,70,72$, $34,36,33,35,75,77,66,42,40,41,43,97,81,73,48,49,71,50,101,79,55$, $76,56,78,104,82,62,80,83,84,85,87,86,88,89,90,92,91,61,94,95,107$, $93,68,99,109,98,74,111,102,96,113,114,100,115,103,116,106,117,105$, $108,110,112,118,119,120$

Bala-Carter Label: $D_{6}\left(a_{1}\right)$

Special

Real form: EVIII

Root vectors for $e: 64,65,67,73,-30,-47,71$

Parabolic: Levi-Type: $A_{5}$

Cartan subalgebra: $44,-52,6,5,4,46,8,-47$

Roots vectors for Levi: $\pm(44,6,5,4,46,51,13,12,53,57,20,59,63,66,97)$

Roots vectors for nilradical: $-52,8,-47,-45,54,-39,-37,-38,60,2,3,-30$, $7,67,10,11,-23,14,15,73,18,19,58,101$, $21,22,-31,26,27,64,65,69,28,29,-24,71,70,72,17,36,-16,76,78,77,25$, $79,-9,82,83,33,104,32,34,35,87,107,40,75,42,43,109,48,80,81,84,-1$, $111,85,86,88,41,89,90,92,49,50,94,96,55,56,113,114,61,62,91,93,68$, $95,98,100,99,115,103,116,106,117,74,102,118,105,108,110,119,120,112$

Bala-Carter Label: $A_{6} \oplus A_{1}$

Real form: EVIII

Root vectors for $e: 64,65,66,67,69,-37,-38$

Parabolic: Levi-Type: $A_{4} \oplus A_{2} \oplus A_{1}$

Cartan subalgebra: $44,52,6,-45,4,2,39,8$

Roots vectors for Levi: $\pm(4,2,39,8,10,46,47,53,54,60), \pm(44,6,51), \pm(52)$

Roots vectors for nilradical: $-45,5,-37,-38,3,13,12,-30,-31,57,11$, $20,18,-24,7,63,17,-23,26,59,14,15,19,69,58,-16$, $66,67,22,27,25,97,65,-9,21,73,33,32,64,101,28,29,40,71,70,72,34$, $36,-1,76,78,75,77,42,48,35,80,83,81,79,82,41,43,86,104,85,87,49$, $50,107,89,90,56,84,55,94,109,88,61,62,111,91,93,68,92,113,98,96$, $95,114,100,99,115,103,116,74,102,106,117,105,118,108,110,112,119,120$ 


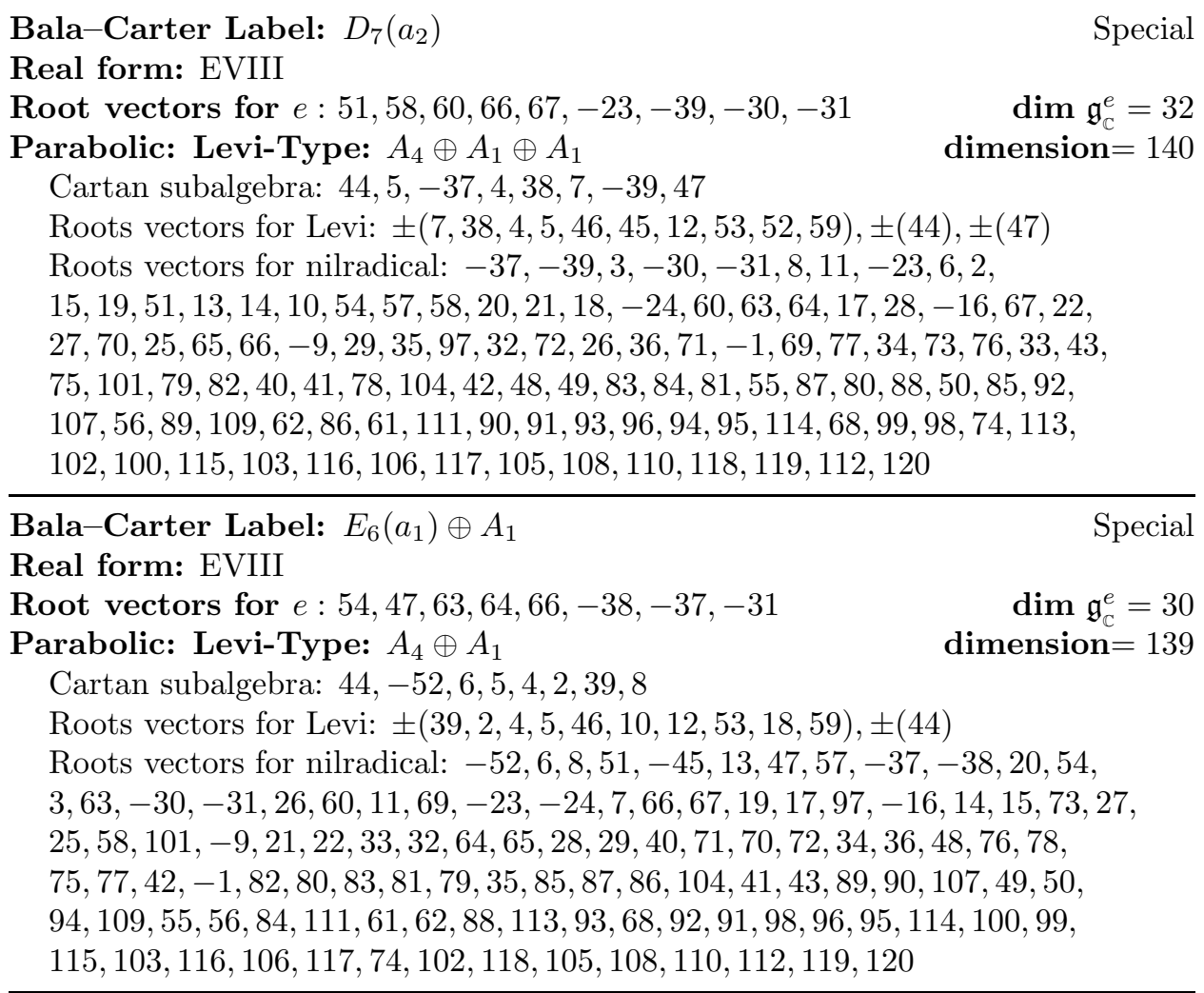

Bala-Carter Label: $E_{7}\left(a_{1}\right)$

Real form: EVIII

Root vectors for $e: 54,57,58,60,-38,-44,-47,-45 \quad \operatorname{dim} \mathfrak{g}_{\mathbb{C}}^{e}=20$

Parabolic: Levi-Type: $A_{3}$

Cartan subalgebra: $-44,-52,51,5,4,46,8,-47$

Roots vectors for Levi: $\pm(5,4,46,12,53,59)$

Roots vectors for nilradical: $-44,-52,51,8,-47,6,-45,57,54,-39,13,3,-38$, $63,60,2,-37,20,11,7,97,67,10,-30,66,19,58,15,101,18,-23,14,73,64,65$, $-31,69,27,21,22,26,70,72,17,71,28,29,-24,77,25,76,78,36,-16,104,32$, $82,83,33,79,-9,75,35,87,107,40,34,81,43,109,48,80,42,84,-1,111,85$, $86,88,41,89,90,92,49,50,94,114,55,56,113,96,93,62,91,61,98,95,68$, $115,99,100,116,103,117,106,102,74,118,105,108,110,119,120,112$

This concludes the proof.

We end the paper with the following theorem.

Theorem 3.10. Maintaining our previous notation, let $\mathfrak{g}_{\mathbb{C}}$ be a simple complex Lie algebra other than $E_{8}$, and fix a nonminimal special nilpotent orbit $\mathcal{O}$ of $G_{\mathbb{C}}$ on $\mathfrak{g}_{\mathbb{C}}$. Then there exists a real form $G$ such that some irreducible component of $\mathcal{O} \cap \mathfrak{p}_{\mathbb{C}}$ is a Richardson orbit of $K_{\mathbb{C}}$ on the nilpotent cone of $\mathfrak{p}_{\mathbb{C}}$.

Proof. If $\mathfrak{g}_{\mathbb{C}}$ is classical, then a proof is given [8]; otherwise, the theorem follows from the propositions above. 


\section{REFERENCES}

1. R. Carter, Simple Groups of Lie Type, Wiley Classics Library, John Wiley \& Sons, London, 1989. MR 1013112 (90g:20001)

2. A. W. Knapp, Lie Groups Beyond an Introduction, Second Edition, Progr. Math., Birkhäuser, Boston, 140 2002. MR.1920389 (2003c:22001)

3. G. Lusztig and N. Spaltenstein, Induced unipotent classes, J. London Math. Soc. 19 (1979), 41-52. MR0527733 (82g:20070)

4. A. G. Noël, Computing theta-stable parabolic subalgebras using LiE, Lecture Notes Comput. Sci., Springer-Verlag 3039 (2004), 335-342.

5. R. W. Richardson, Conjugacy classes in parabolic subgroups of semisimple algebraic groups, Bull. London Math. Soc. 6 (1974), 21-24. MR0330311 (48:8648)

6. J. Sekiguchi, Remarks on real nilpotent orbits of a symmetric pair, J. Math. Soc. Japan 39, No. 1 (1987), 127-138. MR0867991 (88g:53053)

7. P. Tauvel, Quelques résultats sur les algèbres de Lie symétriques, Bull. Sci. Math. 125 No. 8 (2001), 641-665. MR 1872599 (2002j:17008)

8. P. Trapa, Richardson orbits for real classical groups, J. Algebra 286 (2005), 361-385. MR.2128022

9. E. B. Vinberg, On the classification of the nilpotent elements of graded Lie algebras, Dokl. Acad. Nauk SSSR 225 (1975b), 745-748 (Russian). English translation: Soviet Math. Doklady 16 (1975), 1517-1520. MR 0506488 (58:22194)

10. M. A. A. Van Leeuwen, A. M. Cohen, and B. Lisser, LiE A package for Lie Group Computations, Computer Algebra Nederland, Amsterdam, The Netherlands (1992).

11. D. Djoković Classification of nilpotent elements in simple exceptional real Lie algebras of inner type and description of their centralizers, J. Algebra 112 (1987) 577-585. MR0926619 (89b:17010)

12. D. Djoković, Classification of nilpotent elements in simple real Lie algebras $E_{6(6)}$ and $E_{6(-26)}$ and description of their centralizers, J. Algebra 116 (1988) 196-207. MR0944155(89k:17022)

Mathematics Department, The University of Massachusetts, Boston, Massachusetts 02125-3393

E-mail address: anoel@math.umb.edu 